\title{
Synthesis of Novel Halogenated Heterocyclic compounds and their uses as Target SARS-CoV-2 main Protease (Mpro) and Potential Anti-Covid-19
}

Rafat Mohareb ( $\nabla$ raafat_mohareb@yahoo.com )

Cairo University Faculty of Science https://orcid.org/0000-0003-3922-803X

Fahad M. Almutairi

Department of Biotechnology

Abdo A. Elfiky

School of Theoretical Modeling Department of Biophysics

Mahmoud A.A. Mahmoud

University of Tabouk: University of Tabuk

Wagnat W. Wardakhan

National Organization for Drug Control and Research

Mervat S. Mohamed

Cairo University Faculty of Science

Ali Saber Abdelhameed

University of Tabouk: University of Tabuk

\section{Research Article}

Keywords: Halogenated compounds, SARS-CoV-2, Coronavirus, Main protease, Mpro, Molecular Docking

Posted Date: March 13th, 2021

DOl: https://doi.org/10.21203/rs.3.rs-284501/v1

License: (c) (i) This work is licensed under a Creative Commons Attribution 4.0 International License.

Read Full License 


\section{Synthesis of Novel Halogenated Heterocyclic compounds and their uses as Target SARS-CoV-2 main Protease (Mro) and Potential Anti-Covid-19}

Fahad M. Almutairi, ${ }^{\text {a }}$ Rafat M. Mohareb, ${ }^{* b}$ Abdo A. Elfiky, ${ }^{\mathrm{c}}$ Mahmoud A.A. Mahmoud, ${ }^{\text {d }}$ Wagnat W. Wardakhan, ${ }^{\mathrm{e}}$ Mervat S. Mohamed, ${ }^{\mathrm{a}, \mathrm{f}}$ Ali Saber Abdelhameed ${ }^{\mathrm{g}}$

${ }^{* a}$ Department of Biochemistry, Faculty of Science, University of Tabuk, Kingdom of Saudi Arabia.

Tabuk 71491, P. O. Box 741.

${ }^{b}$ Department of Chemistry, Faculty of Science, Cairo University, Giza, Egypt

${ }^{\mathrm{c} B i o p h y s i c s ~ D e p a r t m e n t, ~ F a c u l t y ~ o f ~ S c i e n c e, ~ C a i r o ~ U n i v e r s i t y, ~ G i z a, ~ E g y p t ~}$

${ }^{\mathrm{d}}$ Department of Chemistry, Faculty of Science, University of Tabuk, Kingdom of Saudi Arabia, Tabuk 71491, P. O. Box 741.

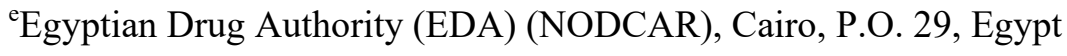

fDepartment of Chemistry, Faculty of Science, University of Tabuk, University of Tabuk, Kingdom of Saudi Arabia

${ }^{g}$ Department of Pharmaceutical Chemistry, College of pharmacy, King Saud University, Riyadh,11451, Kingdom of Saudi Arabia.

Abstract: Since the first appearance of the coronavirus disease-2019 (COVID-19) in Wuhan, China, in December 2019, it has been spreading globally with devastating ramifications. The lack of anti-COVID-19 treatment to date warrants urgent research into potential therapeutic targets. Virtual drug screening techniques enable the identification of novel compounds that are capable of targeting the severe acute respiratory syndrome coronavirus 2 (SARS-CoV-2) main protease $\left(\mathrm{M}^{\mathrm{pro}}\right)$. The latter plays a fundamental role in mediating viral replication and transcription, rendering it an attractive drug target. In this study, twenty six novel halogenated, heterocyclic compounds, which can inhibit $\mathrm{M}^{\text {pro }}$, were tested by molecular docking combined with molecular dynamics simulation. Three compounds showed the highest binding affinity to the protein active site and their binding modes coincide with that of Nelfinavir. The binding of the halogenated compounds to $\mathrm{M}^{\mathrm{pro}}$ may inhibit the replication and transcription of SARS-CoV-2 and, ultimately, stop 
the virallife cycle. In times of dire need for anti-COVID-19 treatment, this study lays the groundwork for further experimental research to investigate the efficacy and potential medical uses of these compounds to treat COVID-19. Novel compounds including fused thiophene, pyrimidine and pyran derivatives were tested against human RNA N7-MTase (hRNMT) and selected viral N7-MTases such as SARS-CoV nsp14 and Vaccinia D1D12 complex to evaluate their specificity and their molecular modeling was also studied in the aim of producing anti covid-19 target molecules.

\section{Keywords:}

Halogenated compounds; SARS-CoV-2; Coronavirus; Main protease; ${ }^{\text {pro }}$; Molecular Docking

\section{Introduction}

Medicinal chemistry had its beginning when chemists, pharmacist and physicians isolated and purified active principles of plants and animals' tissues and taken from microorganism and their fermentation products. Some of these chemicals has been associated with therapeutic properties: Medicinal chemistry which has leaned on the classical fields of chemistry, especially organic chemistry, biology and some area of physics [1-8]. A limited number of natural and synthetic products and serve directly as therapeutic agents although lack of specificity frequently limits their application in human and veterinary medicines and in analogous pesticidal and other uses in agriculture [9-12]. By dissecting the structure of these products chemically, one arrives at its therapeutically significant molecular sections, the pharamacophores, the portion that can be deleted are of no interest as components of drug action; they are regarded as the result of the biosynthetic efforts on the parent organism to construct materials for its own matebolic or defensive purposes. Most of the drugs belong to the class of heterogenius compounds. Heterocyclic compounds played a vital role in the metabolism of all living cells; large number of them are five and six membered heterocyclic compounds having one to three heteroatoms in their nucleus [13-18]. The compounds may be thiophene, pyran derivatives that were basis of genetic material DNA, and these heterocyclic compounds may be isolated or 
fused heterocyclic systems. Some of the common heterocyclic compounds used in the medicines are as amino acids like proline, histidine and tryptophan, the vitamins and coenzymes precursors such as thiamine, riboflavin, pyridoxine, folic acid, biotin, B12 and E families of the vitamins. There is a vast number of pharmacologically active heterocylic compounds, many of which are in regular clinical use. The thiophene and its derivatives have a vital role in biological properties [19-22]. In the present work, we report the synthesis of new heterocyclic compounds with high chloro content together with their studying as potential anti-corona virus. The current pandemic coronavirus disease-2019 (COVID-19) is a new infectious pneumonia-like illness caused by a novel virusstrain, so-called severe acute respiratory syndrome coronavirus 2or SARS-CoV-2 $[23,24]$. The hydroxychloroquine an approved drug for malaria disease by FDA was explored as a medication for SARS-CoV-2 [25,26]. Previous reports revealed that, the chloroquine and hydroxychloroquine can inhibit the coronavirus (COVID-19) by changing the $\mathrm{pH}$ at the surface of the cell membrane. This action can inhibit the attachment of the virus to the cell membrane. In addition, it can prevent nucleic acid replication, glycosylation of viral proteins, virus assembly, new virus particle delivery, virus release, and other mechanisms to obtain its antiviral effects [27]. Heterocycles are widely investigated for possible medicinal applications [28,29]. Favipiravir (1) [30], amodiaquine (2) [31], 20-fluoro-20-deoxycytidine (3) [32], and (4) [33] are known as antiviral drugs. In biological systems, the halogenation of organic molecules is catalyzed by enzymes haloperoxidases such as MPO, EPO and LPO in normal physiological processes, which combine the inorganic substrates $\mathrm{X}^{-}$and $\mathrm{H}_{2} \mathrm{O}_{2}$ to produce RHS. RHS in turn oxidize the hydro-carbon or-nitrogen substrate $\mathrm{RH} / \mathrm{RNH}$ to synthesize many halogenated organic compounds (RX/RNX):

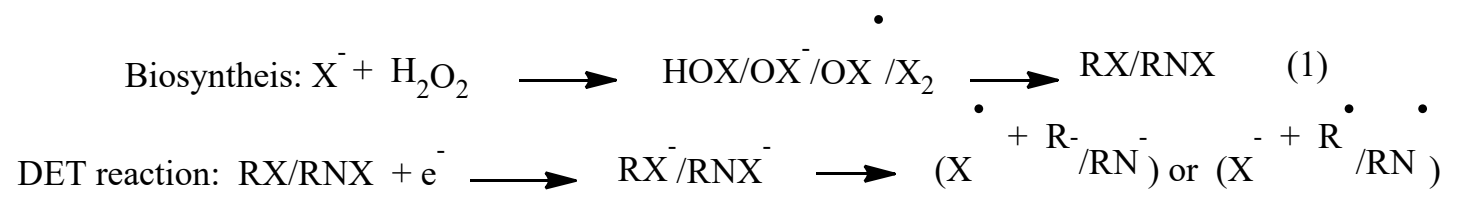

By reaction (1), many halogenated organic compounds are biosynthesize they inhibit various RNA and DNA viruses. For that reason, in the present work we concerned with heterocyclic compounds that are halogen rich and studying their potential anti-covide 19 
inhibitions. Hence, we aim to determine whether the protease of COVID-19 can be a target protein of these nucleotides in silico. Moreover, a comparative study between these drugs with the FDA approved remdesivir and hydrocloroquine antiviral drugs against a broad range of RNA viruses [34] has been established to investigate the effectiveness of the drugs as inhibitors for COVID 19. Demonstrations for the synthesis of halogen rich heterocyclic compounds together with their potentialities for corona virus were demonstrated through this work.

\section{Experimental}

\subsection{General}

The melting points obtained for the synthesized compounds were uncorrected and were recorded using an Electrothermal digital melting point apparatus. IR spectra ( $\mathrm{KBr}$ discs) were measured using a FTIR plus 460 or PyeUnicam SP-1000 spectrophotometer. ${ }^{1} \mathrm{HNMR}$ spectra were measured using Varian Gemini-200 (200 MHz) and Jeol AS $500 \mathrm{MHz}$ instruments spectra were performed in DMSO- $d_{6}$ as solvent using TMS as internal standard and chemical shifts are expressed as $\delta$ ppm. MS (EI) spectra were measured using Hewlett Packard 5988 A GC/MS system and GCMS-QP 1000 Ex Shimadzu instruments. Analytical data were obtained from the Micro-analytical Data Unit at Cairo University and were performed on Vario EL III Elemental analyzer.

\subsubsection{General procedure for the synthesis of the $2,2,2$-trichloroethylidene)- cyclohexane-1,3-dione derivatives $3 a, b$}

To a solution of either $1 \mathrm{a}(1.12 \mathrm{~g}, 0.01 \mathrm{~mol})$ or $1 \mathbf{b}(1.40 \mathrm{~g}, 0.01 \mathrm{~mol})$ in absolute ethanol $(40 \mathrm{~mL}, 0.01 \mathrm{~mol})$ containing triethylamine $(0.50 \mathrm{~mL})$ trichloroacetonitrile $(1.42 \mathrm{~g}, 0.01$ mol) was added. The reaction mixture was heated under reflux for $2 \mathrm{~h}$ then was left to cool and the formed solid product, in each case, was collected by filtration.

\section{2-(1-Amino-2,2,2-trichloroethylidene)cyclohexane-1,3-dione (3a)}


Yellow crystals from ethanol, yield (1.99 g, $78 \%$ ), m.p 204-207 ${ }^{\circ} \mathrm{C}$. IR (KBr) $v$ max $\mathrm{cm}^{-1}: 3472-3346\left(\mathrm{NH}_{2}\right), 1702,1688(2 \mathrm{CO}), 1630(\mathrm{C}=\mathrm{C}) ;{ }^{1} \mathrm{H}$ NMR (DMSO- $d_{6}, 300$ $\mathrm{MHz}): \delta=4.85$ (s, $2 \mathrm{H}, \mathrm{D}_{2} \mathrm{O}$ exchangeable, $\left.\mathrm{NH}_{2}\right), 1.96-1.82\left(\mathrm{~m}, 2 \mathrm{H}, \mathrm{CH}_{2}\right), 2.95-2.80$ (m, $4 \mathrm{H}, 2 \mathrm{CH}_{2}$ ); ${ }^{13} \mathrm{C}$ NMR (DMSO- $\left.d_{6}, 75 \mathrm{MHz}\right): \delta 173.4,168.0$ (C-1, C-3), 112.3, 90.8 (C-2, C-1 ethylidene), 94.8 ( $\left.\mathrm{CCl}_{3}\right), 40.8,38.2,17.1$ (C-4, C-5, C-6), Anal. Calculated for $\mathrm{C}_{8} \mathrm{H}_{8} \mathrm{Cl}_{3} \mathrm{NO}_{2}$ : C, 37.46; H, 3.14; N, 5.46. Found: C, 37.80; H, 3.39; N, 5.52. MS: m/e 256 $\left(\mathrm{M}^{+}, 28 \%\right)$.

\section{2-(1-Amino-2,2,2-trichloroethylidene)-5,5-dimethylcyclohexane-1,3-dione (3b)}

Yellow crystals from ethanol, yield (2.21 g, 78 \%), m.p 233-235 ${ }^{\circ} \mathrm{C}$. IR (KBr) v max $\mathrm{cm}^{-1}:$ 3490-3362 (NH2), 1705, $1689(2 \mathrm{CO}), 1635(\mathrm{C}=\mathrm{C}) ;{ }^{1} \mathrm{H}$ NMR (DMSO-d6, 300 $\mathrm{MHz}): \delta=4.89\left(\mathrm{~s}, 2 \mathrm{H}, \mathrm{D}_{2} \mathrm{O}\right.$ exchangeable, $\left.\mathrm{NH}_{2}\right), 2.96,3.01\left(2 \mathrm{~s}, 4 \mathrm{H}, 2 \mathrm{CH}_{2}\right), 1.09,1.08$ $\left(2 \mathrm{~s}, 6 \mathrm{H}, 2 \mathrm{CH}_{3}\right) ;{ }^{13} \mathrm{C}$ NMR (DMSO- $\left.d_{6}, 75 \mathrm{MHz}\right): \delta 173.1,168.3(\mathrm{C}-1, \mathrm{C}-3), 112.5,90.5$ (C-2, C-1 ethylidene), $94.9\left(\mathrm{CCl}_{3}\right), 42.9,38.1,17.4$ (C-4, C-5, C-6), $24.8\left(2 \mathrm{CH}_{3}\right)$. Anal. Calculated for $\mathrm{C}_{10} \mathrm{H}_{12} \mathrm{Cl}_{3} \mathrm{NO}_{2}$ : C, 42.21; $\mathrm{H}, 4.25 ; \mathrm{N}, 4.92$. Found: $\mathrm{C}, 42.38 ; \mathrm{H}, 4.08 ; \mathrm{N}$, 5.16. MS: $\mathrm{m} / \mathrm{e} 284\left(\mathrm{M}^{+}, 48 \%\right)$.

\subsubsection{General procedure for the synthesis of the 6,7-dihydrobenzo[b]thiophene derivatives 5a-d}

Equimolar amounts of either 3a $(2.54 \mathrm{~g}, 0.01 \mathrm{~mol})$ or $3 \mathbf{b}(2.84 \mathrm{~g}, 0.01 \mathrm{~mol})$ in absolute ethanol $(40 \mathrm{~mL})$ containing triethylamine $(1.50 \mathrm{~mL})$ each of elemental sulfur $(0.32 \mathrm{~g}$, $0.01 \mathrm{~mol})$ and either malononitrile $(0.66 \mathrm{~g}, 0.01 \mathrm{~mol})$ or ethyl cyanoacetate $(1.13 \mathrm{~g}, 0.01$ mol) were added. The reaction mixture, in each case, was heated under reflux for $2 \mathrm{~h}$ then poured onto ice/water mixture containing a few drops of hydrochloric acid and the formed solid product was collected by filtration.

\section{2-Amino-4-(1-amino-2,2,2-trichloroethylidene)-5-oxo-4,5,6,7-tetrahydrobenzo[b]- thiophene-3-carbonitrile (5a)}

Yellow crystals from acetic acid, yield (2.22 g, $66 \%)$, m.p $170-173{ }^{\circ} \mathrm{C}$. IR $(\mathrm{KBr}) v$ max $\mathrm{cm}^{-1}$ : 3496-3352 ( $\left.\mathrm{NH}_{2}\right), 2220(\mathrm{CN}), 1689(\mathrm{CO}), 1632(\mathrm{C}=\mathrm{C}) ;{ }^{1} \mathrm{H}$ NMR (DMSO-d 6,300 $\mathrm{MHz}): \delta=5.25,4.80\left(2 \mathrm{~s}, 4 \mathrm{H}, \mathrm{D}_{2} \mathrm{O}\right.$ exchangeable, $\left.2 \mathrm{NH}_{2}\right), 2.13,2.45\left(2 \mathrm{t}, 4 \mathrm{H}, 2 \mathrm{CH}_{2}\right) ;{ }^{13} \mathrm{C}$ NMR (DMSO- $\left.d_{6}, 75 \mathrm{MHz}\right): \delta 168.2$ (C-5), 132.6, 133.8, 138.0, 140.1 (thiophene C), 
$116.8(\mathrm{CN}), 112.1,90.6$ (C-2, C-1 ethylidene), $94.4\left(\mathrm{CCl}_{3}\right), 40.7,39.6$ (C-3, C-4). Anal. Calculated for $\mathrm{C}_{11} \mathrm{H}_{8} \mathrm{Cl}_{3} \mathrm{~N}_{3} \mathrm{OS}$ : C, 39.25; H, 2.40; N, 12.48; S, 9.53. Found: C, 39.50; H, 2.66; N, 12.72; S, 9.37. MS: m/e $336\left(\mathrm{M}^{+}, 36 \%\right)$.

Ethyl 2-amino-4-(1-amino-2,2,2-trichloroethylidene)-5-oxo-4,5,6,7-tetrahydrobenzo[b]thiophene-3-carboxylate (5b)

Yellow crystals from acetic acid, yield (2.68 g, $70 \%$ ), m.p 170-172 ${ }^{\circ} \mathrm{C}$. IR (KBr) v max $\mathrm{cm}^{-1}$ : 3479-3330 ( $\left.\mathrm{NH}_{2}\right), 1700,1689(2 \mathrm{CO}), 1636(\mathrm{C}=\mathrm{C}) ;{ }^{1} \mathrm{H}$ NMR (DMSO-do, 300 $\mathrm{MHz}): \delta=5.28,4.76\left(2 \mathrm{~s}, 4 \mathrm{H}, \mathrm{D}_{2} \mathrm{O}\right.$ exchangeable, 2NH$), 4.22(\mathrm{q}, 2 \mathrm{H}, J=6.89 \mathrm{~Hz}$, $\left.\mathrm{OCH}_{2} \mathrm{CH}_{3}\right), 2.16,2.49\left(2 \mathrm{t}, 4 \mathrm{H}, 2 \mathrm{CH}_{2}\right), 1.12\left(\mathrm{t}, 3 \mathrm{H}, J=6.89 \mathrm{~Hz}, \mathrm{OCH}_{2} \underline{\mathrm{CH}_{3}}\right) ;{ }^{13} \mathrm{C} \mathrm{NMR}$ (DMSO-d6, 75 MHz): $\delta 168.5$ (C-5), 133.8, 134.5, 138.0, 140.8 (thiophene C), 112.4, 90.2 (C-2, C-1 ethylidene), $94.3\left(\mathrm{CCl}_{3}\right), 50.2\left(\mathrm{OCH}_{2} \mathrm{CH}_{3}\right), 40.9,39.8$ (C-3, C-4), 16.8 $\left(\mathrm{OCH}_{2} \underline{\mathrm{CH}_{3}}\right)$. Anal. Calculated for $\mathrm{C}_{13} \mathrm{H}_{13} \mathrm{Cl}_{3} \mathrm{~N}_{2} \mathrm{O}_{3} \mathrm{~S}$ : C, 40.70; H, 3.42; N, 7.30; S, 8.36. Found: C, 40.63; H, 3.25; N, 7.59; S, 8.42. MS: m/e $383\left(\mathrm{M}^{+}, 42 \%\right)$.

\section{2-Amino-4-(1-amino-2,2,2-trichloroethylidene)-7,7-dimethyl-5-oxo-4,5,6,7-tetra- hydrobenzo $[b]$ thiophene-3-carbonitrile (5c)}

Yellow crystals from acetic acid, yield (2.12 g, 58 \%), m.p $155-157 \quad{ }^{\circ} \mathrm{C}$. IR (KBr) v max $\mathrm{cm}^{-1}$ : 3479-3332 (NH$), 2220(\mathrm{CN}), 1692(\mathrm{CO}), 1631(\mathrm{C}=\mathrm{C}) ;{ }^{1} \mathrm{H}$ NMR (DMSO-d6, 300 $\mathrm{MHz}): \delta=5.28,4.82\left(2 \mathrm{~s}, 4 \mathrm{H}, \mathrm{D}_{2} \mathrm{O}\right.$ exchangeable, $\left.2 \mathrm{NH}_{2}\right), 2.48\left(\mathrm{~s}, 2 \mathrm{H}, \mathrm{CH}_{2}\right), 1.09,1.08$ $\left(2 \mathrm{~s}, 6 \mathrm{H}, 2 \mathrm{CH}_{3}\right) ;{ }^{13} \mathrm{C} \mathrm{NMR}$ (DMSO- $\left.d 6,75 \mathrm{MHz}\right): \delta 168.5$ (C-5), 133.5, 136.3, 137.4, 141.1 (thiophene C), $117.0(\mathrm{CN}), 112.3,90.5$ (C-2, C-1 ethylidene), $94.7\left(\mathrm{CCl}_{3}\right), 40.8,39.8$ (C-3, C-4), $24.6\left(2 \mathrm{CH}_{3}\right)$. Anal. Calculated for $\mathrm{C}_{13} \mathrm{H}_{12} \mathrm{Cl}_{3} \mathrm{~N}_{3} \mathrm{OS}: \mathrm{C}, 42.82 ; \mathrm{H}, 3.32 ; \mathrm{N}$, 11.52; S, 8.79. Found: C, 42.63; H, 3.41; N, 11.27; S, 8.92. MS: m/e $364\left(\mathrm{M}^{+}, 48 \%\right)$.

\section{Ethyl 2-amino-4-(1-amino-2,2,2-trichloroethylidene)-7,7-dimethyl-5-oxo-4,5,6,7- tetrahydrobenzo $[b]$ thiophene-3-carboxylate $(5 \mathrm{~d})$}

Orange crystals from acetic acid, yield (2.80 g, $68 \%$ ), m.p 211-213 ${ }^{\circ} \mathrm{C}$. IR (KBr) v max $\mathrm{cm}^{-1}$ : 3493-3356 (NH2), 1703, 1689 (2CO), $1632(\mathrm{C}=\mathrm{C}) ;{ }^{1} \mathrm{H}$ NMR (DMSO-d6, 200 $\mathrm{MHz}): \delta=5.32,4.73\left(2 \mathrm{~s}, 4 \mathrm{H}, \mathrm{D}_{2} \mathrm{O}\right.$ exchangeable, 2NH$), 4.21(\mathrm{q}, 2 \mathrm{H}, J=7.13 \mathrm{~Hz}$, $\left.\mathrm{OCH}_{2} \mathrm{CH}_{3}\right), 2.52\left(\mathrm{~s}, 2 \mathrm{H}, \mathrm{CH}_{2}\right), 1.11\left(\mathrm{t}, 3 \mathrm{H}, J=7.13 \mathrm{~Hz}, \mathrm{OCH}_{2} \underline{\mathrm{CH}_{3}}\right), 1.09,1.07(2 \mathrm{~s}, 6 \mathrm{H}$, 
$2 \mathrm{CH}_{3}$ ); ${ }^{13} \mathrm{C}$ NMR (DMSO- $\left.d_{6}, 75 \mathrm{MHz}\right): \delta 168.2(\mathrm{C}-5), 132.3,133.9,138.3,140.5$ (thiophene C), 112.6, $90.8\left(\mathrm{C}-2, \mathrm{C}-1\right.$ ethylidene), $94.5\left(\mathrm{CCl}_{3}\right), 50.21\left(\mathrm{OCH}_{2} \mathrm{CH}_{3}\right), 40.6$, $39.5(\mathrm{C}-3, \mathrm{C}-4), 24.6\left(2 \mathrm{CH}_{3}\right), 16.6\left(\mathrm{OCH}_{2} \underline{\mathrm{CH}_{3}}\right)$. Anal. Calculated for $\mathrm{C}_{15} \mathrm{H}_{17} \mathrm{Cl}_{3} \mathrm{~N}_{2} \mathrm{O}_{3} \mathrm{~S}: \mathrm{C}$, 43.76; H, 4.16; N, 6.80; S, 7.79. Found: C, 43.59; H, 4.06; N, 6.93; S, 8.02. MS: m/e 411 $\left(\mathrm{M}^{+}, 31 \%\right)$.

\subsubsection{General procedure for the synthesis of the 4,6-dihydrothieno[3,2- flquinazoline derivatives 7a-d}

To a solution of either $5 \mathbf{a}(3.36 \mathrm{~g}, 0.01 \mathrm{~mol}), \mathbf{5 b}(3.83 \mathrm{~g}, 0.01 \mathrm{~mol}), \mathbf{5 c}(3.62 \mathrm{~g}, 0.01 \mathrm{~mol})$ or $5 \mathrm{~d}(4.11 \mathrm{~g}, 0.01 \mathrm{~mol})$ in ethanol $(40 \mathrm{~mL})$ containing triethylamine $(1.0 \mathrm{~mL})$ phenylisothiocyanate $(1.30 \mathrm{~g}, 0.01 \mathrm{~mol})$ was added and the reaction mixture was heated under reflux for $3 \mathrm{~h}$ then left to cool. The formed solid crystals, in each case, were collected by filtration.

\section{8-Amino-3-mercapto-4-phenyl-1-(trichloromethyl)-4,6-dihydrothieno[3,2- $f$ quinazoline-9-carbonitrile (7a)}

Yellow crystals from acetic acid, yield (3.08 g, $68 \%$ ), m.p233-235 ${ }^{\circ} \mathrm{C}$. IR $(\mathrm{KBr}) v$ max $\mathrm{cm}^{-1}$ : 3483-3361 ( $\left.\mathrm{NH}_{2}\right), 3055\left(\mathrm{CH}\right.$ aromatic), $2220(\mathrm{CN}), 1631(\mathrm{C}=\mathrm{C})$; ${ }^{1} \mathrm{H}$ NMR (DMSO$\left.d_{6}, 300 \mathrm{MHz}\right): \delta=8.20(\mathrm{~s}, 1 \mathrm{H}, \mathrm{SH}), 7.28-7.39\left(\mathrm{~m}, 5 \mathrm{H}, \mathrm{C}_{6} \mathrm{H}_{5}\right), 5.84(\mathrm{t}, 1 \mathrm{H}, \mathrm{CH}), 4.83(\mathrm{~s}$, $2 \mathrm{H}, \quad \mathrm{D}_{2} \mathrm{O}$ exchangeable, $\left.\mathrm{NH}_{2}\right), 2.80\left(\mathrm{~d}, 2 \mathrm{H}, \mathrm{CH}_{2}\right) ;{ }^{13} \mathrm{C}$ NMR (DMSO- $d_{6}, 75$ MHz): $\delta 172.3(\mathrm{C}=\mathrm{N}), 131.4,132.5,138.3,140.6$ (thiophene $\mathrm{C}), 116.9(\mathrm{CN}), 112.1,90.6$ (C-2, C-1 ethylidene), 123.6, 121.6, 119.3, $98.3(2 \mathrm{C}=\mathrm{C}), 94.5\left(\mathrm{CCl}_{3}\right), 40.9,39.8$ (C-3, C4), Anal. Calculated for $\mathrm{C}_{18} \mathrm{H}_{11} \mathrm{Cl}_{3} \mathrm{~N}_{4} \mathrm{~S}_{2}: \mathrm{C}, 47.64 ; \mathrm{H}, 2.44 ; \mathrm{N}, 12.35 ; \mathrm{S}, 14.13$. Found: $\mathrm{C}$, 47.59; H, 2.56; N, 12.62; S, 14.08. MS: m/e $453\left(\mathrm{M}^{+}, 42 \%\right)$.

\section{Ethyl 8-amino-3-mercapto-4-phenyl-1-(trichloromethyl)-4,6-dihydrothieno[3,2- $f$ quinazoline-9-carboxylate (7b)}

Yellow crystals from acetic acid, yield (3.51 g, $70 \%$ ), m.p $142-144{ }^{\circ} \mathrm{C}$. IR (KBr) v max $\mathrm{cm}^{-1}$ : 3479-3330 ( $\left.\mathrm{NH}_{2}\right), 3050\left(\mathrm{CH}\right.$ aromatic), $1689(\mathrm{CO}), 1636(\mathrm{C}=\mathrm{C})$; ${ }^{1} \mathrm{H}$ NMR (DMSO$\left.d_{6}, 300 \mathrm{MHz}\right): \delta=8.20(\mathrm{~s}, 1 \mathrm{H}, \mathrm{SH}), 7.28-7.39\left(\mathrm{~m}, 5 \mathrm{H}, \mathrm{C}_{6} \mathrm{H}_{5}\right), 5.84(\mathrm{~s}, 1 \mathrm{H}, \mathrm{CH}), 4.83(\mathrm{~s}$, $2 \mathrm{H}, \mathrm{D}_{2} \mathrm{O}$ exchangeable, $\left.\mathrm{NH}_{2}\right), 4.20$ (q, $\left.2 \mathrm{H}, J=5.90 \mathrm{~Hz}, \mathrm{OCH}_{2} \mathrm{CH}_{3}\right), 2.80\left(\mathrm{~d}, 2 \mathrm{H}, \mathrm{CH}_{2}\right.$ ), $1.13\left(\mathrm{t}, 3 \mathrm{H}, J=5.90 \mathrm{~Hz}, \mathrm{OCH}_{2} \underline{\mathrm{CH}_{3}}\right) ;{ }^{13} \mathrm{C} \mathrm{NMR}$ (DMSO- $\left.d 6,75 \mathrm{MHz}\right): \delta 172.1(\mathrm{C}=\mathrm{N})$, 
165.2 (CO), 131.4, 132.5, 138.3, 140.6 (thiophene C), $116.9(\mathrm{CN}), 112.1,90.6$ (C-2, C-1 ethylidene), 123.6, 121.3, 120.5, $99.6(2 \mathrm{C}=\mathrm{C}), 94.2\left(\mathrm{CCl}_{3}\right), 50.3\left(\mathrm{OCH}_{2} \mathrm{CH}_{3}\right), 40.5,39.4$ (C-3, C-4), $16.3\left(\mathrm{OCH}_{2} \underline{\mathrm{CH}_{3}}\right)$. Anal. Calculated for $\mathrm{C}_{20} \mathrm{H}_{16} \mathrm{Cl}_{3} \mathrm{~N}_{3} \mathrm{O}_{2} \mathrm{~S}_{2}$ : C, 47.96; H, 3.22; N, 8.39; S, 12.80.Found: C, 47.70; H, 3.52; N, 8.56; S, 12.62. MS: m/e 500 (M+2 $28 \%)$.

\section{8-Amino-3-mercapto-6,6-dimethyl-4-phenyl-1-(trichloromethyl)-4,6-dihydrothieno-} $[3,2-f]$ quinazoline-9-carbonitrile (7c)

Yellow crystals from acetic acid, yield (3.63 g, $70 \%)$, m.p $142-144{ }^{\circ} \mathrm{C}$. IR ( $\left.\mathrm{KBr}\right) v$ max $\mathrm{cm}^{-1}$ : 3479-3330 $\left(\mathrm{NH}_{2}\right), 3050\left(\mathrm{CH}\right.$ aromatic), $1636(\mathrm{C}=\mathrm{C}) ;{ }^{1} \mathrm{H}$ NMR (DMSO- $d 6,300$ $\mathrm{MHz}): \delta=8.22(\mathrm{~s}, 1 \mathrm{H}, \mathrm{SH}), 7.28-7.42\left(\mathrm{~m}, 5 \mathrm{H}, \mathrm{C}_{6} \mathrm{H}_{5}\right), 5.60(\mathrm{~s}, 1 \mathrm{H}, \mathrm{CH}), 4.85\left(\mathrm{~s}, 2 \mathrm{H}, \mathrm{D}_{2} \mathrm{O}\right.$ exchangeable, $\mathrm{NH}_{2}$ ), $1.09,1.06$ (s, $\left.6 \mathrm{H}, 2 \mathrm{CH}_{3}\right) ;{ }^{13} \mathrm{C}$ NMR (DMSO- $d_{6}, 75 \mathrm{MHz}$ ): $\delta 172.3$ $(\mathrm{C}=\mathrm{N}), 131.6,134.8,138.7,140.8$ (thiophene $\mathrm{C}), 116.7(\mathrm{CN}), 112.3,90.8(\mathrm{C}-2, \mathrm{C}-1$ ethylidene), 124.4, 121.1, 120.3, $99.8(2 \mathrm{C}=\mathrm{C}), 94.4\left(\mathrm{CCl}_{3}\right), 40.2,39.6(\mathrm{C}-3, \mathrm{C}-4), 24.5$ $\left(2 \mathrm{CH}_{3}\right)$. Anal. Calculated for $\mathrm{C}_{20} \mathrm{H}_{15} \mathrm{Cl}_{3} \mathrm{~N}_{4} \mathrm{~S}_{2}$ : C, 49.85; H, 3.14; N, 11.63; S, 13.31 . Found: $\mathrm{C}, 49.72 ; \mathrm{H}, 3.28 ; \mathrm{N}, 11.80 ; \mathrm{S}, 13.27$. MS: m/e $381\left(\mathrm{M}^{+}, 60 \%\right)$.

\section{Ethyl 8-amino-3-mercapto-6,6-dimethyl-4-phenyl-1-(trichloromethyl)-4,6- dihydrothieno[3,2-f]quinazoline-9-carboxylate (7d)}

Orange crystals from acetic acid, yield (3.17 g, $60 \%)$, m.p 188-190 ${ }^{\circ} \mathrm{C}$. IR (KBr) v max $\mathrm{cm}^{-1}$ : 3479-3337 $\left(\mathrm{NH}_{2}\right), 1688(\mathrm{CO}), 1630(\mathrm{C}=\mathrm{C}) ;{ }^{1} \mathrm{H}$ NMR (DMSO- $\left.d_{6}, 300 \mathrm{MHz}\right): \delta=$ $8.23(\mathrm{~s}, 1 \mathrm{H}, \mathrm{SH}), 7.26-7.42\left(\mathrm{~m}, 5 \mathrm{H}, \mathrm{C}_{6} \mathrm{H}_{5}\right), 5.86(\mathrm{~s}, 1 \mathrm{H}, \mathrm{CH}), 4.81\left(\mathrm{~s}, 2 \mathrm{H}, \mathrm{D}_{2} \mathrm{O}\right.$ exchangeable, $\mathrm{NH}_{2}$ ), 4.22 (q, $\left.2 \mathrm{H}, J=6.14 \mathrm{~Hz}, \underline{\mathrm{OCH}}_{2} \mathrm{CH}_{3}\right), 1.12$ (t, $3 \mathrm{H}, J=6.14 \mathrm{~Hz}$, $\left.\mathrm{OCH}_{2} \underline{\mathrm{CH}_{3}}\right), 1.09,1.07\left(2 \mathrm{~s}, 6 \mathrm{H}, 2 \mathrm{CH}_{3}\right) ;{ }^{13} \mathrm{C}$ NMR (DMSO- $\left.d_{6}, 75 \mathrm{MHz}\right): \delta 172.3(\mathrm{C}=\mathrm{N})$, 164.8 (CO), 131.1, 133.8, 137.9, 140.3 (thiophene C), $116.8(\mathrm{CN}), 112.0,90.8$ (C-2, C-1 ethylidene), 123.3, 121.6, 120.6, $99.8(2 \mathrm{C}=\mathrm{C}), 94.3\left(\mathrm{CCl}_{3}\right), 50.1\left(\mathrm{OCH}_{2} \mathrm{CH}_{3}\right), 40.6,39.2$ (C-3, C-4), $24.7\left(2 \mathrm{CH}_{3}\right), 16.2\left(\mathrm{OCH}_{2} \mathrm{CH}_{3}\right)$. Anal. Calculated for $\mathrm{C}_{22} \mathrm{H}_{20} \mathrm{Cl}_{3} \mathrm{~N}_{3} \mathrm{O}_{2} \mathrm{~S}_{2}: \mathrm{C}$, 49.96; H, 3.81; N, 7.94; S, 12.13. Found: C, 49.77; H, 3.62; N, 8.03; S, 12.35. MS: m/e $528\left(\mathrm{M}^{+}, 46 \%\right)$.

2.1.4. General procedure for the synthesis of the 4,6-dihydrothieno[3,2flquinazolin-1-ol derivatives 8a-d 
To solution of either 7a $(4.53 \mathrm{~g}, 0.01 \mathrm{~mol}), 7 \mathbf{b}(5.00 \mathrm{~g}, 0.01 \mathrm{~mol}), 7 \mathbf{c}(3.81 \mathrm{~g}, 0.01 \mathrm{~mol})$ or $7 \mathbf{d}(5.28 \mathrm{~g}, 0.01 \mathrm{~mol})$ in ethanol $(70 \mathrm{~mL})$ containing sodium hydroxide solution $(5 \mathrm{~mL}, 10$ $\%$ )was heated under reflux for $6 \mathrm{~h}$. the solid product, in each case, produced upon pouring onto ice/water mixture containing a few drops of hydrochloric acid (till $\mathrm{pH}$ 6) was collected by filtration.

\section{8-Amino-1-hydroxy-3-mercapto-4-phenyl-4,6-dihydrothieno[3,2-f]quinazoline-9- carbonitrile (8a)}

Yellow crystals from 1,4-dioxane, yield (2.46 g, $70 \%)$, m.p210-212 ${ }^{\circ} \mathrm{C}$. IR $(\mathrm{KBr}) v$ max $\mathrm{cm}^{-1}$ : 3563-3349 (OH, NH$), 3053\left(\mathrm{CH}\right.$ aromatic), $2220(\mathrm{CN}), 1633(\mathrm{C}=\mathrm{C}) ;{ }^{1} \mathrm{H}$ NMR (DMSO- $d_{6}, 300 \mathrm{MHz}$ ): $\delta=10.21$ (s, $1 \mathrm{H}, \mathrm{D}_{2} \mathrm{O}$ exchangeable, $\mathrm{OH}$ ), 8.22 (s, $1 \mathrm{H}, \mathrm{SH}$ ), 7.24$7.40\left(\mathrm{~m}, 5 \mathrm{H}, \mathrm{C}_{6} \mathrm{H} 5\right), 5.82(\mathrm{t}, 1 \mathrm{H}, \mathrm{CH}), 4.86\left(\mathrm{~s}, 2 \mathrm{H}, \mathrm{D}_{2} \mathrm{O}\right.$ exchangeable, $\left.\mathrm{NH}_{2}\right), 2.82(\mathrm{~d}, 2 \mathrm{H}$, $\left.\mathrm{CH}_{2}\right) ;{ }^{13} \mathrm{C}$ NMR (DMSO- $\left.d 6,75 \mathrm{MHz}\right): \delta 172.1(\mathrm{C}=\mathrm{N}), 130.2,133.6,138.1,141.3$ (thiophene C), $116.8(\mathrm{CN}), 112.3,90.4$ (C-2, C-1 ethylidene), 123.2, 121.8, 119.1, 98.0 $(2 \mathrm{C}=\mathrm{C}), 40.6,39.7$ (C-3, C-4), Anal. Calculated for $\mathrm{C}_{17} \mathrm{H}_{12} \mathrm{~N}_{4} \mathrm{OS}_{2}$ : C, 57.93; H, 3.43; N, 15.90; S, 18.20. Found: C, 57.73; H, 3.29; N, 16.17; S, 18.06. MS: m/e $352\left(\mathrm{M}^{+}, 36 \%\right)$.

Ethyl 8-amino-1-hydroxy-3-mercapto-4-phenyl-4,6-dihydrothieno[3,2-f]quinazoline9-carboxylate (8b)

Yellow crystals from 1,4-dioxane, yield (2.64 g, 66 \%), m.p 159-161 ${ }^{\circ} \mathrm{C}$. IR (KBr) v $\max \mathrm{cm}^{-1}$ : 3569-3346 (OH, NH 2$), 3052\left(\mathrm{CH}\right.$ aromatic), $1702(\mathrm{CO}), 1636(\mathrm{C}=\mathrm{C}) ;{ }^{1} \mathrm{H}$ NMR (DMSO-d6, $300 \mathrm{MHz}): \delta=10.21\left(\mathrm{~s}, 1 \mathrm{H}, \mathrm{D}_{2} \mathrm{O}\right.$ exchangeable, $\left.\mathrm{OH}\right), 8.22(\mathrm{~s}, 1 \mathrm{H}$, $\mathrm{SH})$, 7.24-7.43 (m, 5H, $\left.\mathrm{C}_{6} \mathrm{H}_{5}\right), 5.86(\mathrm{~s}, 1 \mathrm{H}, \mathrm{CH}), 4.89\left(\mathrm{~s}, 2 \mathrm{H}, \mathrm{D}_{2} \mathrm{O}\right.$ exchangeable, $\left.\mathrm{NH}_{2}\right)$, 4.22 (q, $\left.2 \mathrm{H}, J=6.89 \mathrm{~Hz}, \mathrm{OCH}_{2} \mathrm{CH}_{3}\right), 2.82$ (d, $\left.2 \mathrm{H}, \mathrm{CH}_{2}\right), 1.12$ (t, $3 \mathrm{H}, J=6.89 \mathrm{~Hz}$, $\left.\mathrm{OCH}_{2} \underline{\mathrm{CH}_{3}}\right) ;{ }^{13} \mathrm{C}$ NMR (DMSO-d $\left.6,75 \mathrm{MHz}\right): \delta 172.3(\mathrm{C}=\mathrm{N}), 166.3(\mathrm{CO}), 131.6,132.3$, 138.2, 140.4 (thiophene C), 116.7 (CN), 112.3, 90.5 (C-2, C-1 ethylidene), 123.3, 121.6, 120.3, $99.8(2 \mathrm{C}=\mathrm{C}), 50.2\left(\mathrm{OCH}_{2} \mathrm{CH}_{3}\right), 40.8,39.6(\mathrm{C}-3, \mathrm{C}-4), 16.1\left(\mathrm{OCH}_{2} \underline{\mathrm{CH}_{3}}\right)$. Anal. Calculated for $\mathrm{C}_{19} \mathrm{H}_{17} \mathrm{~N}_{3} \mathrm{O}_{3} \mathrm{~S}_{2}: \mathrm{C}, 57.12 ; \mathrm{H}, 4.29 ; \mathrm{N}, 10.52 ; \mathrm{S}, 16.05$.Found: $\mathrm{C}, 57.08 ; \mathrm{H}$, 4.38; N, 10.38; S, 15.83. MS: m/e $399\left(\mathrm{M}^{+}, 32 \%\right)$.

\section{8-Amino-1-hydroxy-3-mercapto-6,6-dimethyl-4-phenyl-4,6-dihydrothieno[3,2-} $f$ quinazoline-9-carbonitrile (8c) 
Yellow crystals from ethanol, yield $(2.74 \mathrm{~g}, 72 \%)$, m.p 189-192 ${ }^{\circ} \mathrm{C}$. IR (KBr) v max $\mathrm{cm}^{-1}$ : 3569-3342 (OH, $\left.\mathrm{NH}_{2}\right), 3050\left(\mathrm{CH}\right.$ aromatic), $1638(\mathrm{C}=\mathrm{C})$; ${ }^{1} \mathrm{H}$ NMR (DMSO-d6, $300 \mathrm{MHz}): \delta=10.30$ (s, 1H, $\mathrm{D}_{2} \mathrm{O}$ exchangeable, $\mathrm{OH}$ ), 8.25 (s, 1H, SH), 7.26-7.39 (m, $\left.5 \mathrm{H}, \mathrm{C}_{6} \mathrm{H}_{5}\right), 5.62(\mathrm{~s}, 1 \mathrm{H}, \mathrm{CH}), 4.83\left(\mathrm{~s}, 2 \mathrm{H}, \mathrm{D}_{2} \mathrm{O}\right.$ exchangeable, $\left.\mathrm{NH}_{2}\right), 1.09,1.07(\mathrm{~s}, 6 \mathrm{H}$, $\left.2 \mathrm{CH}_{3}\right) ;{ }^{13} \mathrm{C}$ NMR (DMSO- $\left.d_{6}, 75 \mathrm{MHz}\right): \delta 172.1(\mathrm{C}=\mathrm{N}), 130.8,133.2,139.6,142.3$ (thiophene C), $116.9(\mathrm{CN}), 112.1,90.5$ (C-2, C-1 ethylidene), 123.5, 122.8, 120.1, 99.5 $(2 \mathrm{C}=\mathrm{C}), 40.5,39.4(\mathrm{C}-3, \mathrm{C}-4), 24.64\left(2 \mathrm{CH}_{3}\right)$. Anal. Calculated for $\mathrm{C}_{19} \mathrm{H}_{16} \mathrm{~N}_{4} \mathrm{OS}_{2}: \mathrm{C}$, 59.98; H, 4.24; N, 14.73; S, 16.85. Found: C, 59.88; H, 4.52; N, 14.80; S, 16.69. MS: m/e $380\left(\mathrm{M}^{+}, 56 \%\right)$.

\section{Ethyl 8-amino-1-hydroxy-3-mercapto-6,6-dimethyl-4-phenyl-4,6-dihydrothieno[3,2- flquinazoline-9-carboxylate (8d)}

Pale yellow crystals from ethanol, yield (2.65g, $62 \%)$, m.p 211-213 ${ }^{\circ} \mathrm{C}$. IR $(\mathrm{KBr}) v$ max $\mathrm{cm}^{-1}$ : 3459-3341 ( $\left.\mathrm{NH}_{2}\right), 1702(\mathrm{CO}), 1632(\mathrm{C}=\mathrm{C}) ;{ }^{1} \mathrm{H}$ NMR (DMSO- $\left.d_{6}, 300 \mathrm{MHz}\right): \delta=$ $10.28\left(\mathrm{~s}, 1 \mathrm{H}, \mathrm{D}_{2} \mathrm{O}\right.$ exchangeable $\left.\mathrm{OH}\right), 8.26(\mathrm{~s}, 1 \mathrm{H}, \mathrm{SH}), 7.24-7.46\left(\mathrm{~m}, 5 \mathrm{H}, \mathrm{C}_{6} \mathrm{H}_{5}\right), 5.85$ (s, $1 \mathrm{H}, \mathrm{CH}), 4.83$ (s, $2 \mathrm{H}, \mathrm{D}_{2} \mathrm{O}$ exchangeable, $\left.\mathrm{NH}_{2}\right), 4.23$ (q, $2 \mathrm{H}, J=5.80 \mathrm{~Hz}, \mathrm{OCH}_{2} \mathrm{CH}_{3}$ ), $1.12\left(\mathrm{t}, 3 \mathrm{H}, J=5.80 \mathrm{~Hz}, \mathrm{OCH}_{2} \mathrm{CH}_{3}\right), 1.08,1.06\left(2 \mathrm{~s}, 6 \mathrm{H}, 2 \mathrm{CH}_{3}\right) ;{ }^{13} \mathrm{C}$ NMR (DMSO- $d 6,75$ MHz): $\delta 172.2(\mathrm{C}=\mathrm{N}), 165.8(\mathrm{CO}), 131.3,133.5,137.5,141.6$ (thiophene $\mathrm{C}), 116.7(\mathrm{CN})$, 112.1, 90.6 (C-2, C-1 ethylidene), 123.2, 121.8, 120.4, $99.6(2 \mathrm{C}=\mathrm{C}), 50.4\left(\mathrm{OCH}_{2} \mathrm{CH}_{3}\right)$, $40.8, \quad 39.4(\mathrm{C}-3, \mathrm{C}-4), 24.6\left(2 \mathrm{CH}_{3}\right), 16.3\left(\mathrm{OCH}_{2} \mathrm{CH}_{3}\right)$. Anal. Calculated for $\mathrm{C}_{21} \mathrm{H}_{21} \mathrm{~N}_{3} \mathrm{O}_{3} \mathrm{~S}_{2}$ : C, 58.99; H, 4.95; N, 9.83; S, 15.00. Found: C, 58.82; H, 5.21; N, 9.02; S, 14.87. MS: $\mathrm{m} / \mathrm{e} 427\left(\mathrm{M}^{+}, 436 \%\right)$.

\subsubsection{General procedures for the synthesis of the 8,9-dihydro-4H-thieno[2,3- g]chromene derivatives 10a-m}

To a solution of either compound $\mathbf{5 a}$ (3.36 g, $0.01 \mathrm{~mol})$, or $\mathbf{5 c}$ (3.64 g, $0.01 \mathrm{~mol})$ in 1,4dioxane $(40 \mathrm{~mL})$ containing triethylamine $(1.00 \mathrm{~mL})$ each of either benzaldehyde $(1.08 \mathrm{~g}$, $0.01 \mathrm{~mol}), 4$-chlorobenzaldehyde $(1.40 \mathrm{~g}, 0.01 \mathrm{~mol})$ or 4-methoxybenzaldehyde $(1.37 \mathrm{~g}$, $0.01 \mathrm{~mol})$ and either malononitrile $(0.66 \mathrm{~g}, 0.01 \mathrm{~mol})$ or ethyl cyanoacetate $(1.13 \mathrm{~g}, 0.01$ mol) was added. The reaction mixture, in each case, was heated under reflux for $1 \mathrm{~h}$ then poured onto ice/water containing a few drops of hydrochloric acid and the formed solid product was collected by filtration. 


\section{2,6-Diamino-4-(1-amino-2,2,2-trichloroethylidene)-8-phenyl-8,9-dihydro-4H-}

thieno[2,3-g]chromene-3,7-dicarbonitrile(10a)

Pale brown crystals from 1,4-dioxane, yield (3.19 g, 65 \%), m.p 166-168 ${ }^{\circ} \mathrm{C}$. IR (KBr) v $\max \mathrm{cm}^{-1}: 3459-3346\left(\mathrm{NH}_{2}\right), 3050\left(\mathrm{CH}\right.$ aromatic), 2223, $2220(2 \mathrm{CN}), 1636(\mathrm{C}=\mathrm{C}) ;{ }^{1} \mathrm{H}$ NMR (DMSO-d6, 300 MHz): $\delta=7.25-7.42$ (m, 5H, C6 $\mathrm{H}_{5}$ ), 5.90 (s, 1H, pyran H-4), 4.80, 5.22, $5.39\left(3 \mathrm{~s}, 6 \mathrm{H}, \mathrm{D}_{2} \mathrm{O}\right.$ exchangeable, $\left.3 \mathrm{NH}_{2}\right), 2.80\left(\mathrm{~s}, 2 \mathrm{H}, \mathrm{CH}_{2}\right) ;{ }^{13} \mathrm{C}$ NMR (DMSO-d6, $75 \mathrm{MHz}): \delta 120.1,123.5,125.4,128.5,130.8,133.7,137.9,138.3,139.0,140.2,142.5$, $143.8\left(\mathrm{C}_{6} \mathrm{H}_{5}\right.$, thiophene, pyran C), 116.9, $117.3(2 \mathrm{CN}), 112.4,90.2$ (C-2, C-1 ethylidene), $94.2\left(\mathrm{CCl}_{3}\right), 53.8\left(\mathrm{CH}_{2}\right)$. Anal. Calculated for $\mathrm{C}_{21} \mathrm{H}_{14} \mathrm{Cl}_{3} \mathrm{~N}_{5} \mathrm{OS}: \mathrm{C}, 51.39 ; \mathrm{H}, 2.88 ; \mathrm{N}$, 14.27; S, 6.53. Found: C, 51.59; H, 3.11; N, 14.62; S, 6.80. MS: m/e 490 (M+, $32 \%)$.

Ethyl 2,6-diamino-4-(1-amino-2,2,2-trichloroethylidene)-3-cyano-8-phenyl-8,9dihydro-4H-thieno[2,3-g]chromene-7-carboxylate (10b)

Yellow crystals from 1,4-dioxane, yield (3.76 g, $70 \%$, m.p 210-212 ${ }^{\circ} \mathrm{C}$. IR (KBr) v $\max \mathrm{cm}^{-1}$ : 3472-3348 (NH2), $3052(\mathrm{CH}$ aromatic), $2220(\mathrm{CN}), 1689(\mathrm{CO}), 1632(\mathrm{C}=\mathrm{C})$; ${ }^{1} \mathrm{H}$ NMR (DMSO-d6, $300 \mathrm{MHz}$ ): $\delta=7.32-7.45$ (m, 5H, $\left.\mathrm{C}_{6} \mathrm{H}_{5}\right), 5.92$ (s, 1H, pyran H-4), 5.22, 5.39, $4.83\left(3 \mathrm{~s}, 6 \mathrm{H}, \mathrm{D}_{2} \mathrm{O}\right.$ exchangeable, $\left.3 \mathrm{NH}_{2}\right), 4.22$ (q, $2 \mathrm{H}, J=7.22 \mathrm{~Hz}$, $\left.\mathrm{OCH}_{2} \mathrm{CH}_{3}\right), 2.82\left(\mathrm{~s}, 2 \mathrm{H}, \mathrm{CH}_{2}\right), 1.12\left(\mathrm{t}, 3 \mathrm{H}, J=7.22 \mathrm{~Hz}, \mathrm{OCH}_{2} \mathrm{CH}_{3}\right) ;{ }^{13} \mathrm{C}$ NMR (DMSO$\left.d_{6,} 75 \mathrm{MHz}\right): \delta 165.9$ (CO), 120.6, 123.4, 125.2, 128.6, 131.4, 132.8, 136.2, 138.5, 139.3, 140.5, 142.1, 142.9 ( $\mathrm{C}_{6} \mathrm{H}_{5}$, thiophene, pyran C), $116.7(\mathrm{CN}), 112.5,90.5(\mathrm{C}-2, \mathrm{C}-1$ ethylidene), $94.5\left(\mathrm{CCl}_{3}\right), 53.8\left(\mathrm{CH}_{2}\right), 50.2\left(\underline{\mathrm{OCH}}_{2} \mathrm{CH}_{3}\right), 16.8\left(\mathrm{OCH}_{2} \underline{\mathrm{CH}}_{3}\right)$. Anal. Calculated for $\mathrm{C}_{23} \mathrm{H}_{19} \mathrm{Cl}_{3} \mathrm{~N}_{4} \mathrm{O}_{3} \mathrm{~S}$ : C, 51.36; H, 3.56; N, 10.42; S, 5.96. Found: C, 51.49; H, 3.43; N, 10.28; S, 6.03. MS: m/e $537\left(\mathrm{M}^{+}, 38 \%\right)$.

\section{2,6-Diamino-4-(1-amino-2,2,2-trichloroethylidene)-9,9-dimethyl-8-phenyl-8,9-} dihydro-4H-thieno[2,3-g]chromene-3,7-dicarbonitrile (10c)

Pale brown crystals from 1,4-dioxane, yield (3.52 g, 68 \%), m.p 201-203 ${ }^{\circ} \mathrm{C}$. IR (KBr) v $\max \mathrm{cm}^{-1}$ : 3473-3342 $\left(\mathrm{NH}_{2}\right), 3054\left(\mathrm{CH}\right.$ aromatic), 2222-2220 $(2 \mathrm{CN}), 1632(\mathrm{C}=\mathrm{C}) ;{ }^{1} \mathrm{H}$ NMR (DMSO-d $d_{6}, 300$ MHz): $\delta=7.27-7.40$ (m, 5H, $\mathrm{C}_{6} \mathrm{H}_{5}$ ), 5.93 (s, 1H, pyran H-4), 4.82, 5.21, $5.42\left(3 \mathrm{~s}, 6 \mathrm{H}, \mathrm{D}_{2} \mathrm{O}\right.$ exchangeable, $\left.3 \mathrm{NH}_{2}\right), 1.09,1.07\left(2 \mathrm{~s}, 6 \mathrm{H}, 2 \mathrm{CH}_{3}\right) ;{ }^{13} \mathrm{C} \mathrm{NMR}$ (DMSO-d6, 75 MHz): $\delta 120.3,120.6,125.4,128.8,130.8,133.7,137.9,138.3,139.0$, 
140.2, 142.5, $143.8\left(\mathrm{C}_{6} \mathrm{H}_{5}\right.$, thiophene, pyran C), 116.9, $117.3(2 \mathrm{CN}), 112.4,90.2$ (C-2, C1 ethylidene), $94.2\left(\mathrm{CCl}_{3}\right), 24.8\left(2 \mathrm{CH}_{3}\right)$. Anal. Calculated for $\mathrm{C}_{23} \mathrm{H}_{18} \mathrm{Cl}_{3} \mathrm{~N}_{5} \mathrm{OS}$ : C, 53.24; H, 3.50; N, 13.50; S, 6.18. Found: C, 53.39; H, 3.42; N, 13.69; S, 6.23. MS: m/e $518\left(\mathrm{M}^{+}\right.$, $36 \%)$.

Ethyl 2-amino-4-(1-amino-2,2,2-trichloroethylidene)-3,7-dicyano-9,9-dimethyl-8phenyl-8,9-dihydro-4H-thieno[2,3-g]chromene-6-carboxylate (10d)

Yellow crystals from 1,4-dioxane, yield (3.68 g, $65 \%$ ), m.p 230-233 ${ }^{\circ} \mathrm{C}$. IR ( $\left.\mathrm{KBr}\right) v$ max cm-1: 3469-3338 $\left(\mathrm{NH}_{2}\right), 3055(\mathrm{CH}$ aromatic), $2220(\mathrm{CN}), 1688(\mathrm{CO}), 1630(\mathrm{C}=\mathrm{C})$; ${ }^{1} \mathrm{H}$ NMR (DMSO- $\left.d_{6}, 300 \mathrm{MHz}\right): \delta=7.28-7.39\left(\mathrm{~m}, 5 \mathrm{H}, \mathrm{C}_{6} \mathrm{H}_{5}\right), 5.68(\mathrm{~s}, 1 \mathrm{H}$, pyran $\mathrm{H}-4)$, 5.33, $4.86\left(2 \mathrm{~s}, 4 \mathrm{H}, \mathrm{D}_{2} \mathrm{O}\right.$ exchangeable, $\left.2 \mathrm{NH}_{2}\right), 4.23\left(\mathrm{q}, 2 \mathrm{H}, J=6.70 \mathrm{~Hz}, \underline{\mathrm{OCH}_{2}} \mathrm{CH}_{3}\right)$, 1.14 (t, $\left.3 \mathrm{H}, J=6.70 \mathrm{~Hz}, \mathrm{OCH}_{2} \mathrm{CH}_{3}\right), 1.09,1.07\left(2 \mathrm{~s}, 6 \mathrm{H}, 2 \mathrm{CH}_{3}\right) ;{ }^{13} \mathrm{C}$ NMR (DMSO-d 6,75 MHz): $\delta 164.8$ (CO), 119.8, 120.2, 125.6, 128.4, 130.3, 132.5, 134.8, 138.9, 139.1, 140.2, 141.5, $142.6\left(\mathrm{C}_{6} \mathrm{H}_{5}\right.$, thiophene, pyran C), $116.9(\mathrm{CN}), 112.1,90.3$ (C-2, C-1 ethylidene), $94.2\left(\mathrm{CCl}_{3}\right), 50.3\left(\mathrm{OCH}_{2} \mathrm{CH}_{3}\right), 24.8\left(2 \mathrm{CH}_{3}\right), 16.7\left(\mathrm{OCH}_{2} \underline{\mathrm{CH}}_{3}\right)$. Anal. Calculated for $\mathrm{C}_{26} \mathrm{H}_{21} \mathrm{Cl}_{3} \mathrm{~N}_{4} \mathrm{O}_{3} \mathrm{~S}$ : C, 54.22; H, 3.68; N, 9.73; S, 5.57. Found: C, 54.59; H, 3.87; N, 9.73; S, 5.80. MS: m/e $575\left(\mathrm{M}^{+}, 68 \%\right)$.

\section{2,6-Diamino-4-(1-amino-2,2,2-trichloroethylidene)-8-(4-chlorophenyl)-8,9-dihydro- $4 H$-thieno[2,3-g]chromene-3,7-dicarbonitrile (10e)}

Pale orange crystals from 1,4-dioxane, yield (3.72 g,71\%), m.p 240-243 ${ }^{\circ} \mathrm{C}$. IR (KBr) v $\operatorname{max~cm}{ }^{-1}: 3468-3373\left(\mathrm{NH}_{2}\right), 3050\left(\mathrm{CH}\right.$ aromatic), 2222, $2220(2 \mathrm{CN}), 1632(\mathrm{C}=\mathrm{C}) ;{ }^{1} \mathrm{H}$ NMR (DMSO- $d_{6}, 300 \mathrm{MHz}$ ): $\delta=7.29-7.52$ (m, 4H, $\mathrm{C}_{6} \mathrm{H}_{4}$ ), 5.73 (s, 1H, pyran H-4), 4.83, 5.20, $5.36\left(3 \mathrm{~s}, 6 \mathrm{H}, \mathrm{D}_{2} \mathrm{O}\right.$ exchangeable, $3 \mathrm{NH}_{2}$ ), $2.78\left(\mathrm{~s}, 2 \mathrm{H}, \mathrm{CH}_{2}\right) ;{ }^{13} \mathrm{C}$ NMR (DMSO- $d_{6}$, $75 \mathrm{MHz}): \delta 119.3,120.4,122.6,127.9,130.5,132.8,136.6,138.6,139.4,140.6,142.7$, $143.4\left(\mathrm{C}_{6} \mathrm{H}_{4}\right.$, thiophene, pyran $\left.\mathrm{C}\right), 116.9,117.1(2 \mathrm{CN}), 112.4,90.3$ (C-2, C-1 ethylidene), $94.2\left(\mathrm{CCl}_{3}\right), 53.1\left(\mathrm{CH}_{2}\right)$. Anal. Calculated for $\mathrm{C}_{2} \mathrm{H}_{13} \mathrm{Cl}_{4} \mathrm{~N}_{5} \mathrm{OS}: \mathrm{C}, 48.02 ; \mathrm{H}, 2.49 ; \mathrm{N}$, 13.33; S, 6.10. Found: C, 47.92; H, 2.60; N, 13.52; S, 6.26. MS: m/e $525\left(\mathrm{M}^{+}, 46 \%\right)$.

Ethyl 2,6-diamino-4-(1-amino-2,2,2-trichloroethylidene)-8-(4-chlorophenyl)-3cyano-8,9-dihydro-4H-thieno[2,3-g]chromene-7-carboxylate (10f) 
Yellow crystals from 1,4-dioxane, yield (3.43 g, $60 \%$ ), m.p 155-157 $\quad{ }^{\circ} \mathrm{C}$. IR (KBr) v max cm-1: 3484-3346 $\left(\mathrm{NH}_{2}\right), 3050(\mathrm{CH}$ aromatic), $2220(\mathrm{CN}), 1690(\mathrm{CO}), 1630(\mathrm{C}=\mathrm{C})$; ${ }^{1} \mathrm{H}$ NMR (DMSO- $\left.d_{6}, 300 \mathrm{MHz}\right): \delta=7.26-7.53\left(\mathrm{~m}, 4 \mathrm{H}, \mathrm{C}_{6} \mathrm{H}_{4}\right), 5.64(\mathrm{~s}, 1 \mathrm{H}$, pyran $\mathrm{H}-4)$, $5.38,5.33,4.84\left(3 \mathrm{~s}, 6 \mathrm{H}, \mathrm{D}_{2} \mathrm{O}\right.$ exchangeable, $\left.3 \mathrm{NH}_{2}\right), 4.21(\mathrm{q}, 2 \mathrm{H}, J=5.88 \mathrm{~Hz}$, $\mathrm{OCH}_{2} \mathrm{CH}_{3}$ ), $2.90\left(\mathrm{~s}, 2 \mathrm{H}, \mathrm{CH}_{2}\right), 1.14$ (t, $3 \mathrm{H}, J=5.88 \mathrm{~Hz}, \mathrm{OCH}_{2} \underline{\mathrm{CH}_{3}}$ ); ${ }^{13} \mathrm{C}$ NMR (DMSO$\left.d_{6,}, 75 \mathrm{MHz}\right): \delta 165.4$ (CO), 120.4, 120.7, 124.7, 126.9, 130.2, 131.9, 134.6, 137.5, 139.1, 140.3, 141.7, $142.8\left(\mathrm{C}_{6} \mathrm{H}_{5}\right.$, thiophene, pyran $\left.\mathrm{C}\right), 116.7(\mathrm{CN}), 112.0,90.1$ (C-2, C-1 ethylidene), $94.6\left(\mathrm{CCl}_{3}\right), 50.1\left(\mathrm{OCH}_{2} \mathrm{CH}_{3}\right), 16.8\left(\mathrm{OCH}_{2} \underline{\mathrm{CH}}_{3}\right)$. Anal. Calculated for $\mathrm{C}_{23} \mathrm{H}_{18} \mathrm{Cl}_{4} \mathrm{~N}_{4} \mathrm{O}_{3} \mathrm{~S}$ : C, 48.27; H, 3.17; N, 9.79; S, 5.60. Found: C, 48.49; H, 3.42; N, 9.86; S, 5.73. MS: $\mathrm{m} / \mathrm{e} 572\left(\mathrm{M}^{+}, 59 \%\right)$.

\section{2,6-Diamino-4-(1-amino-2,2,2-trichloroethylidene)-8-(4-chlorophenyl)-9,9-dimethyl- 8,9-dihydro-4H-thieno[2,3-g]chromene-3,7-dicarbonitrile (10g)}

Pale brown crystals from 1,4-dioxane, yield (4.04g, 73 \%), m.p 177-179 ${ }^{\circ} \mathrm{C}$. IR (KBr) v max cm${ }^{-1}: 3486-3339\left(\mathrm{NH}_{2}\right), 3050\left(\mathrm{CH}\right.$ aromatic), 2222, $2220(2 \mathrm{CN}), 1634(\mathrm{C}=\mathrm{C}) ;{ }^{1} \mathrm{H}$ NMR (DMSO- $d_{6}, 300 \mathrm{MHz}$ ): $\delta=7.26-7.53$ (m, 4H, $\mathrm{C}_{6} \mathrm{H}_{4}$ ), 5.63 (s, $1 \mathrm{H}$, pyran H-4), 4.86, $5.23,5.40\left(3 \mathrm{~s}, 6 \mathrm{H}, \mathrm{D}_{2} \mathrm{O}\right.$ exchangeable, $\left.3 \mathrm{NH}_{2}\right), 1.08,1.07\left(2 \mathrm{~s}, 6 \mathrm{H}, 2 \mathrm{CH}_{3}\right) ;{ }^{13} \mathrm{C} \mathrm{NMR}$ (DMSO-d6, 75 MHz): $\delta 120.4,122.7,124.9,127.3,130.4,132.6,137.7,138.6,139.3$, 140.2, 141.9, $143.4\left(\mathrm{C}_{6} \mathrm{H}_{5}\right.$, thiophene, pyran C), 116.9, $117.3(2 \mathrm{CN}), 112.3,90.6(\mathrm{C}-2, \mathrm{C}-$ 1 ethylidene), $94.5\left(\mathrm{CCl}_{3}\right), 24.6\left(2 \mathrm{CH}_{3}\right)$. Anal. Calculated for $\mathrm{C}_{23} \mathrm{H}_{17} \mathrm{Cl}_{4} \mathrm{~N}_{5} \mathrm{OS}$ : C, 49.93; H, 3.10; N, 12.66; S, 5.80. Found: C, 49.69; H, 3.36; N, 12.72; S, 6.03. MS: m/e $553\left(\mathrm{M}^{+}\right.$, $48 \%)$.

Ethyl 2,6-diamino-4-(1-amino-2,2,2-trichloroethylidene)-8-(4-chlorophenyl)-3cyano-9,9-dimethyl-8,9-dihydro-4H-thieno[2,3-g]chromene-7-carboxylate (10h)

Pale yellow crystals from1,4-dioxane, yield (3.72 g, $62 \%)$, m.p 170-172 ${ }^{\circ} \mathrm{C}$. IR (KBr) v max cm cm $^{-1}$ 3473-3328 (NH2), $3050(\mathrm{CH}$ aromatic), $2220(\mathrm{CN}), 1689(\mathrm{CO}), 1630(\mathrm{C}=\mathrm{C})$; ${ }^{1} \mathrm{H}$ NMR (DMSO- $\left.d_{6}, 300 \mathrm{MHz}\right): \delta=7.23-7.56\left(\mathrm{~m}, 4 \mathrm{H}, \mathrm{C}_{6} \mathrm{H}_{4}\right), 5.62(\mathrm{~s}, 1 \mathrm{H}$, pyran $\mathrm{H}-4)$, 5.36, 5.33, $4.84\left(3 \mathrm{~s}, 6 \mathrm{H}, \mathrm{D}_{2} \mathrm{O}\right.$ exchangeable, $\left.3 \mathrm{NH}_{2}\right), 4.22(\mathrm{q}, 2 \mathrm{H}, J=6.25 \mathrm{~Hz}$, $\left.\mathrm{OCH}_{2} \mathrm{CH}_{3}\right), 1.14$ (t, $\left.3 \mathrm{H}, J=6.26 \mathrm{~Hz}, \mathrm{OCH}_{2} \underline{\mathrm{CH}_{3}}\right), 1.08,1.06\left(\mathrm{~s}, 6 \mathrm{H}, 2 \mathrm{CH}_{3}\right) ;{ }^{13} \mathrm{C} \mathrm{NMR}$ (DMSO-d6, 75 MHz): $\delta 165.1$ (CO), 120.2, 122.8, 124.4, 127.3, 130.6, 132.7, 134.6, 
135.8, 139.3, 140.1, 141.9, $142.6\left(\mathrm{C}_{6} \mathrm{H}_{5}\right.$, thiophene, pyran $\left.\mathrm{C}\right), 116.8(\mathrm{CN}), 112.3,90.3$ (C-2, C-1 ethylidene), $94.5\left(\mathrm{CCl}_{3}\right), 50.3\left(\mathrm{OCH}_{2} \mathrm{CH}_{3}\right), 24.8\left(2 \mathrm{CH}_{3}\right), 16.5\left(\mathrm{OCH}_{2} \underline{\mathrm{CH}}_{3}\right)$. Anal. Calculated for $\mathrm{C}_{25} \mathrm{H}_{22} \mathrm{Cl}_{4} \mathrm{~N}_{4} \mathrm{O}_{3} \mathrm{~S}$ : C, 50.02; H, 3.69; N, 9.33; S, 5.34. Found: C, 50.22; H, 3.76; N, 9.25; S, 5.52. MS: m/e $600\left(\mathrm{M}^{+}, 33 \%\right)$.

\section{2,6-Diamino-4-(1-amino-2,2,2-trichloroethylidene)-8-(4-methoxyphenyl)-8,9-} dihydro-4H-thieno[2,3-g]chromene-3,7-dicarbonitrile (10i)

Pale orange crystals from 1,4-dioxane, yield (3.28 g, $63 \%$ ), m.p195-197 ${ }^{\circ} \mathrm{C}$. IR (KBr) v $\max \mathrm{cm}^{-1}$ : 3487-3353 $\left(\mathrm{NH}_{2}\right), 3050\left(\mathrm{CH}\right.$ aromatic), 2223, $2220(2 \mathrm{CN}), 1630(\mathrm{C}=\mathrm{C}) ;{ }^{1} \mathrm{H}$ NMR (DMSO- $d_{6}, 300 \mathrm{MHz}$ ): $\delta=7.26-7.48\left(\mathrm{~m}, 4 \mathrm{H}, \mathrm{C}_{6} \mathrm{H}_{4}\right), 5.76$ (s, $1 \mathrm{H}$, pyran $\left.\mathrm{H}-4\right), 4.88$, 5.24, 5.38 (3s, 6H, $\mathrm{D}_{2} \mathrm{O}$ exchangeable, $\left.3 \mathrm{NH}_{2}\right), 3.67$ (s, 3H, $\left.\mathrm{OCH}_{3}\right), 2.76\left(\mathrm{~s}, 2 \mathrm{H}, \mathrm{CH}_{2}\right)$; ${ }^{13} \mathrm{C}$ NMR (DMSO-d $6,75 \mathrm{MHz}$ ): $\delta$ 120.3, 121.6, 122.8, 126.4, 129.6, 133.2, 134.8, 137.4, 139.1, 140.8, 141.9, $142.8\left(\mathrm{C}_{6} \mathrm{H}_{4}\right.$, thiophene, pyran $\left.\mathrm{C}\right), 116.7,117.3(2 \mathrm{CN}), 112.6,90.1$ (C-2, C-1 ethylidene), $94.5\left(\mathrm{CCl}_{3}\right), 53.3\left(\mathrm{CH}_{2}\right), 50.1\left(\mathrm{OCH}_{3}\right)$. Anal. Calculated for $\mathrm{C}_{22} \mathrm{H}_{16} \mathrm{Cl}_{4} \mathrm{~N}_{5} \mathrm{O}_{2} \mathrm{~S}: \mathrm{C}, 50.73 ; \mathrm{H}, 3.10 ; \mathrm{N}, 13.45 ; \mathrm{S}, 6.16$. Found: $\mathrm{C}, 50.84 ; \mathrm{H}, 3.35 ; \mathrm{N}$, 13.60; S, 6.32. MS: m/e $520\left(\mathrm{M}^{+}, 64 \%\right)$.

Ethyl 2,6-diamino-4-(1-amino-2,2,2-trichloroethylidene)-3-cyano-8-(4-methoxyphenyl)-8,9-dihydro-4H-thieno[2,3-g]chromene-7-carboxylate (10k)

Yellow crystals from 1,4-dioxane, yield (4.20 g, $74 \%$ ), m.p 205-208 ${ }^{\circ} \mathrm{C}$. IR (KBr) v $\max \mathrm{cm}^{-1}$ : 3469-3352 $\left(\mathrm{NH}_{2}\right), 3050(\mathrm{CH}$ aromatic), $2220(\mathrm{CN}), 1687(\mathrm{CO}), 1630(\mathrm{C}=\mathrm{C})$; ${ }^{1} \mathrm{H}$ NMR (DMSO-d6, $\left.300 \mathrm{MHz}\right): \delta=7.29-7.44\left(\mathrm{~m}, 4 \mathrm{H}, \mathrm{C}_{6} \mathrm{H}_{4}\right), 5.66$ (s, $1 \mathrm{H}$, pyran H-4), 5.34, 5.36, $4.89\left(3 \mathrm{~s}, 6 \mathrm{H}, \mathrm{D}_{2} \mathrm{O}\right.$ exchangeable, $\left.3 \mathrm{NH}_{2}\right), 4.22(\mathrm{q}, 2 \mathrm{H}, J=7.16 \mathrm{~Hz}$, $\left.\mathrm{OCH}_{2} \mathrm{CH}_{3}\right), 3.69$ (s, $\left.3 \mathrm{H}, \mathrm{OCH}_{3}\right), 2.93\left(\mathrm{~s}, 2 \mathrm{H}, \mathrm{CH}_{2}\right), 1.13$ (t, $\left.3 \mathrm{H}, J=7.16 \mathrm{~Hz}, \mathrm{OCH}_{2} \underline{\mathrm{CH}_{3}}\right)$; ${ }^{13} \mathrm{C}$ NMR (DMSO- $\left.d_{6}, 75 \mathrm{MHz}\right): \delta 164.8(\mathrm{CO}), 120.2,120.3,123.8,125.6,130.6,132.4$, 134.7, 136.2, 139.4, 140.9, 141.3, $142.6\left(\mathrm{C}_{6} \mathrm{H}_{5}\right.$, thiophene, pyran C), $116.8(\mathrm{CN}), 112.2$, 90.3 (C-2, C-1 ethylidene), $94.6\left(\mathrm{CCl}_{3}\right), 50.6\left(\mathrm{OCH}_{3}\right), 50.2\left(\mathrm{OCH}_{2} \mathrm{CH}_{3}\right), 16.5$ $\left(\mathrm{OCH}_{2} \underline{\mathrm{CH}}_{3}\right)$. Anal. Calculated for $\mathrm{C}_{24} \mathrm{H}_{21} \mathrm{Cl}_{3} \mathrm{~N}_{4} \mathrm{O}_{4} \mathrm{~S}: \mathrm{C}, 50.76 ; \mathrm{H}, 3.73 ; \mathrm{N}, 9.87 ; \mathrm{S}, 5.65$. Found: C, 50.53; H, 3.59; N, 9.72; S, 5.79. MS: m/e $567\left(\mathrm{M}^{+}, 62 \%\right)$.

\section{2,6-Diamino-4-(1-amino-2,2,2-trichloroethylidene)-8-(4-methoxyphenyl)-9,9-} dimethyl-8,9-dihydro-4H-thieno[2,3-g]chromene-3,7-dicarbonitrile (101) 
Pale brown crystals from 1,4-dioxane, yield (4.01g, $73 \%$ ), m.p 159-161 ${ }^{\circ} \mathrm{C}$. IR (KBr) v $\max \mathrm{cm}^{-1}$ : 3495-3342 $\left(\mathrm{NH}_{2}\right), 3050\left(\mathrm{CH}\right.$ aromatic), 2222, $2220(2 \mathrm{CN}), 1631(\mathrm{C}=\mathrm{C}) ;{ }^{1} \mathrm{H}$ NMR (DMSO- $d_{6}, 300 \mathrm{MHz}$ ): $\delta=7.28-7.56\left(\mathrm{~m}, 4 \mathrm{H}, \mathrm{C}_{6} \mathrm{H}_{4}\right), 5.61$ (s, $1 \mathrm{H}$, pyran H-4), 4.89, 5.08, $5.43\left(3 \mathrm{~s}, 6 \mathrm{H}, \mathrm{D}_{2} \mathrm{O}\right.$ exchangeable, $\left.3 \mathrm{NH}_{2}\right), 3.70\left(\mathrm{~s}, 3 \mathrm{H}, \mathrm{OCH}_{3}\right), 1.08,1.06(2 \mathrm{~s}, 6 \mathrm{H}$, $\left.2 \mathrm{CH}_{3}\right) ;{ }^{13} \mathrm{C}$ NMR (DMSO- $\left.66,75 \mathrm{MHz}\right): \delta 120.2,123.8,124.6,127.9,129.2,132.8,138.3$, $138.9,139.1,140.6,141.7,142.2\left(\mathrm{C}_{6} \mathrm{H}_{5}\right.$, thiophene, pyran C), 116.7, $117.0(2 \mathrm{CN}), 112.1$, 90.3 (C-2, C-1 ethylidene), $94.2\left(\mathrm{CCl}_{3}\right), 50.6\left(\mathrm{OCH}_{3}\right), 24.8\left(2 \mathrm{CH}_{3}\right)$. Anal. Calculated for $\mathrm{C}_{24} \mathrm{H}_{20} \mathrm{Cl}_{3} \mathrm{~N}_{5} \mathrm{O}_{2} \mathrm{~S}: \mathrm{C}, 52.52 ; \mathrm{H}, 3.67 ; \mathrm{N}, 12.76 ; \mathrm{S}, 5.84$. Found: C, 52.62; H, 3.49; N, 12.82; S, 5.72. MS: m/e $548\left(\mathrm{M}^{+}, 50 \%\right)$.

\section{Ethyl 2,6-diamino-4-(1-amino-2,2,2-trichloroethylidene)-3-cyano-8-(4-methoxy- phenyl)-9,9-dimethyl-8,9-dihydro-4H-thieno[2,3-g]chromene-7-carboxylate (10m)}

Orange crystals from1,4-dioxane, yield (4.17g, $70 \%)$, m.p 211-213 ${ }^{\circ} \mathrm{C}$. IR ( $\left.\mathrm{KBr}\right) v$ max $\mathrm{cm}^{-1}$ : 3484-3362 $\left(\mathrm{NH}_{2}\right), 3050\left(\mathrm{CH}\right.$ aromatic), $2220(\mathrm{CN}), 1688(\mathrm{CO}), 1630(\mathrm{C}=\mathrm{C}) ;{ }^{1} \mathrm{H}$ NMR (DMSO- $\left.d_{6}, 300 \mathrm{MHz}\right): \delta=7.28-7.49\left(\mathrm{~m}, 4 \mathrm{H}, \mathrm{C}_{6} \mathrm{H}_{4}\right), 5.68(\mathrm{~s}, 1 \mathrm{H}$, pyran H-4), 5.34, $5.33,4.88\left(3 \mathrm{~s}, 6 \mathrm{H}, \mathrm{D}_{2} \mathrm{O}\right.$ exchangeable, $\left.3 \mathrm{NH}_{2}\right), 4.21$ (q, $\left.2 \mathrm{H}, J=6.80 \mathrm{~Hz}, \mathrm{OCH}_{2} \mathrm{CH}_{3}\right)$, $3.70\left(\mathrm{~s}, 3 \mathrm{H}, \mathrm{OCH}_{3}\right), 1.13$ (t, $\left.3 \mathrm{H}, J=6.80 \mathrm{~Hz}, \mathrm{OCH}_{2} \mathrm{CH}_{3}\right), 1.09,1.07$ (s, $\left.6 \mathrm{H}, 2 \mathrm{CH}_{3}\right) ;{ }^{13} \mathrm{C}$ NMR (DMSO- $\left.d_{6}, 75 \mathrm{MHz}\right): \delta 165.3$ (CO), 120.4, 121.6, 123.6, 128.6, 130.2, 132.9, 134.3, 135.8, 139.1, 140.6, 141.7, $142.8\left(\mathrm{C}_{6} \mathrm{H}_{5}\right.$, thiophene, pyran C), $116.7(\mathrm{CN}), 112.1$, 90.2 (C-2, C-1 ethylidene), $94.3\left(\mathrm{CCl}_{3}\right), 50 . .6\left(\mathrm{OCH}_{3}\right), 50.1\left(\mathrm{OCH}_{2} \mathrm{CH}_{3}\right), 24.6\left(2 \mathrm{CH}_{3}\right)$, $16.3\left(\mathrm{OCH}_{2} \underline{\mathrm{CH}_{3}}\right)$. Anal. Calculated for $\mathrm{C}_{26} \mathrm{H}_{25} \mathrm{Cl}_{3} \mathrm{~N}_{4} \mathrm{O}_{4} \mathrm{~S}: \mathrm{C}, 52.40 ; \mathrm{H}, 4.23 ; \mathrm{N}, 9.40 ; \mathrm{S}$, 5.38. Found: C, 52.62; H, 4.48; N, 9.16; S, 5.73. MS: m/e 595 ( $\left.\mathrm{M}^{+}, 66 \%\right)$.

\subsection{RNA methyltransferase activity assays}

Compounds 3a-10m were tested for their ability to inhibit the methylation of the RNA cap structure. The inhibition induced by each compound $(50 \mu \mathrm{M})$ was determined by a radioactive MTase (methyl transferase) assay (filter binding assay) which consists in measuring the $[3 H]$ radiolabeled methyl transferred from the methyl donor SAM onto RNA substrate (GpppAC4) synthetized by using T7 primase [35]. Compounds 3a-10m designed as mimics of the transition state of RNA 2'-O-methylation were screened against several viral RNA 2'-OMTases from SARS-CoV (nsp10/nsp16 complex), Zika, 
West-Nile, Dengue, Vaccinia (VP39) viruses. At the same time, the compounds were tested against human RNA N7-MTase (hRNMT) and selected viral N7-MTases such as SARS-CoV nsp14 and Vaccinia D1-D12 complex to evaluate their specificity (Table 1). Unexpectedly, all the bisubstrate compounds were barely active against the 2'- $O$ MTases of flaviviruses or coronavirus SARS-CoV. In contrast, most of the compounds displayed inhibition of N7-MTases (methyl transferase).

Table 1. Screening for inhibitory activity of sinefungin and compounds $\mathbf{3 a - 1 0 m}$ at $50 \mu \mathrm{M}$ on N7-MTases.

\begin{tabular}{|c|c|c|c|c|c|c|c|}
\hline Compd & R & X & Y & X' & \multicolumn{3}{|c|}{ \% of inihibition at $50 \mu \mathrm{M}(\%)^{\mathrm{a}}$} \\
& & & & & \multicolumn{3}{|c|}{ SARs-Cov nsp 14 Vaccinia Virus } \\
\hline $\mathbf{3 a}$ & $\mathrm{H}$ & - & - & - & $11.6 \pm 2.3$ & $22.2 \pm 3.1$ & $18.4 \pm 1.6$ \\
\hline $\mathbf{3 b}$ & $\mathrm{CH}_{3}$ & - & - & - & $18.6 \pm 2.8$ & $16.1 \pm 2.8$ & $12.5 \pm 2.5$ \\
\hline $\mathbf{5 a}$ & $\mathrm{H}$ & $\mathrm{CN}$ & - & - & $37.2 \pm 4.1$ & $42.2 \pm 2.6$ & $90.2 \pm 4.5$ \\
\hline $\mathbf{5 b}$ & $\mathrm{H}$ & $\mathrm{COOEt}$ & - & - & $18.7 \pm 2.5$ & $28.3 \pm 2.8$ & $32.3 \pm 4.2$ \\
\hline $\mathbf{5 c}$ & $\mathrm{CH}_{3}$ & $\mathrm{CN}$ & - & - & $48.3 \pm 5.2$ & $50.6 \pm 3.6$ & $46.5 \pm 3.8$ \\
\hline $\mathbf{5 d}$ & $\mathrm{CH}_{3}$ & $\mathrm{COOEt}$ & - & - & $32.5 \pm 2.6$ & $38.6 \pm 3.7$ & $14.3 \pm 1.8$ \\
\hline $\mathbf{7 a}$ & $\mathrm{H}$ & $\mathrm{CN}$ & - & - & $38.2 \pm 2.6$ & $90.1 \pm 3.5$ & $92.4 \pm 2.5$ \\
\hline $\mathbf{7 b}$ & $\mathrm{H}$ & $\mathrm{COOEt}$ & - & & $28.1 \pm 3.5$ & $29.4 \pm 1.8$ & $32.1 \pm 2.4$ \\
\hline $\mathbf{7 c}$ & $\mathrm{CH}_{3}$ & $\mathrm{CN}$ & - & - & $38.1 \pm 3.1$ & $44.2 \pm 3.9$ & $35.3 \pm 3.6$ \\
\hline $\mathbf{7 d}$ & $\mathrm{CH}_{3}$ & $\mathrm{COOEt}$ & - & - & $29.1 \pm 2.6$ & $50.2 \pm 2.9$ & $59.3 \pm 3.4$ \\
\hline $\mathbf{8 a}$ & $\mathrm{H}$ & $\mathrm{CN}$ & -- & - & $54.3 \pm 3.8$ & $89.2 \pm 4.7$ & $38.5 \pm 2.8$ \\
\hline $\mathbf{8 b}$ & $\mathrm{H}$ & $\mathrm{COOEt}$ & - & - & $40.2 \pm 2.9$ & $58.8 \pm 3.4$ & $88.3 \pm 2.4$ \\
\hline $\mathbf{8 c}$ & $\mathrm{CH}_{3}$ & $\mathrm{CN}$ & - & - & $33.2 \pm 2.9$ & $38.1 \pm 3.0$ & $42.5 \pm 2.6$ \\
\hline $\mathbf{8 d}$ & $\mathrm{CH}_{3}$ & $\mathrm{COOEt}$ & - & - & $68.1 \pm 4.8$ & $53.2 \pm 3.6$ & $46.3 \pm 3.4$ \\
\hline $\mathbf{1 0 a}$ & $\mathrm{H}$ & $\mathrm{CN}$ & $\mathrm{H}$ & $\mathrm{CN}$ & $89.3 \pm 5.1$ & $76.2 \pm 3.6$ & $82.6 \pm 5.0$ \\
\hline $\mathbf{1 0 b}$ & $\mathrm{H}$ & $\mathrm{CN}$ & $\mathrm{H}$ & $\mathrm{COOEt}$ & $93.2 \pm 6.3$ & $90.2 \pm 8.9$ & $96.3 \pm 5.4$ \\
\hline $\mathbf{1 0 c}$ & $\mathrm{CH}_{3}$ & $\mathrm{CN}$ & $\mathrm{H}$ & $\mathrm{CN}$ & $96.3 \pm 5.6$ & $90.4 \pm 5.8$ & $92.6 \pm 3.8$ \\
\hline
\end{tabular}




\begin{tabular}{|c|c|c|c|c|c|c|c|}
\hline $\mathbf{1 0 d}$ & $\mathrm{CH}_{3}$ & $\mathrm{CN}$ & $\mathrm{H}$ & $\mathrm{COOEt}$ & $78.3 \pm 6.1$ & $87.2 \pm 4.9$ & $73.4 \pm 4.2$ \\
\hline $\mathbf{1 0 e}$ & $\mathrm{H}$ & $\mathrm{CN}$ & $\mathrm{Cl}$ & $\mathrm{CN}$ & $96.2 \pm 5.8$ & $95.1 \pm 6.2$ & $38.2 \pm 3.9$ \\
\hline $\mathbf{1 0 f}$ & $\mathrm{H}$ & $\mathrm{CN}$ & $\mathrm{Cl}$ & $\mathrm{COOEt}$ & $94.6 \pm 6.8$ & $97.4 \pm 7.2$ & $46.9 \pm 6.4$ \\
\hline $\mathbf{1 0 g}$ & $\mathrm{CH}_{3}$ & $\mathrm{CN}$ & $\mathrm{Cl}$ & $\mathrm{CN}$ & $97.2 \pm 5.3$ & $95.1 \pm 6.3$ & $28.5 \pm 5.8$ \\
\hline $\mathbf{1 0 h}$ & $\mathrm{CH}_{3}$ & $\mathrm{CN}$ & $\mathrm{Cl}$ & $\mathrm{COOEt}$ & $92.8 \pm 4.6$ & $96.3 \pm 5.8$ & $55.6 \pm 4.3$ \\
\hline $\mathbf{1 0}$ & $\mathrm{H}$ & $\mathrm{CN}$ & $\mathrm{OCH}_{3}$ & $\mathrm{CN}$ & $38.2 \pm 2.6$ & $32.4 \pm 4.2$ & $92.6 \pm 5.3$ \\
\hline $\mathbf{1 0 k}$ & $\mathrm{H}$ & $\mathrm{CN}$ & $\mathrm{OCH}_{3}$ & $\mathrm{COOEt}$ & $26.8 \pm 4.6$ & $36.5 \pm 3.2$ & $28.5 \pm 3.8$ \\
\hline $\mathbf{1 0 1}$ & $\mathrm{CH}$ & $\mathrm{CN}$ & $\mathrm{OCH}_{3}$ & $\mathrm{CN}$ & $29.6 \pm 3.1$ & $85.8 \pm 4.8$ & $32.7 \pm 3.6$ \\
\hline $\mathbf{1 0 m}$ & $\mathrm{CH}$ & $\mathrm{CN}$ & $\mathrm{OCH}_{3}$ & $\mathrm{COOEt}$ & $31.3 \pm 3.9$ & $32.4 \pm 4.1$ & $28.6 \pm 3.6$ \\
\hline Sinefungin & - & - & - & - & $98.3 \pm 0.2$ & $99.8 \pm 0.1$ & $99.8 \pm 0.2$ \\
\hline
\end{tabular}

${ }^{a}$ Values are the mean of three independent experiments. The MTase activity was measured using a filter binding assay. Assays were carried out in reaction mixture [40 mM Tris- $\mathrm{HCl}(\mathrm{pH} 8.0), 1 \mathrm{mM}$ DTT, $1 \mathrm{mM}$ $\mathrm{MgCl}_{2}, 2 \mu \mathrm{M}$ SAM and $\left.0.1 \mu \mathrm{M} 3 \mathrm{H}-\mathrm{SAM}\right]$ in the presence of $0.7 \mu \mathrm{M}$ GpppAC4 synthetic RNA and incubated at $30{ }^{\circ} \mathrm{C}$. SARS-CoVnsp14 (50 nM), vaccinia virus capping enzyme (D1-D12) (41 U), human RNA N7, MTase (hRNMT) (50 nM). Compounds were previously dissolved in 100\% DMSO. n.i: no inhibition detected at $50 \mu \mathrm{M}$.

It is clear from Table 1 that $10 a, 10 b, 10 c, 10 e, 10 f, 10 \mathrm{~g}$ and $10 \mathrm{~h}$ showed high \% inhibitions against SARs-Cov nsp 14 with values 89.3, 93.2, 96.3, 96.2, 94.6, 97.2 and 92.8, respectively. On the other hand, compounds $7 \mathbf{a}, 8 \mathbf{8}, 10 \mathrm{~d}, 10 \mathrm{e}, 10 \mathrm{f}, 10 \mathrm{~g}, 10 \mathrm{~h}$ and $10 \mathrm{I}$ revealed high activity on Vaccinia Virus D1-D12 with \% of inhibitions 90.1, 89.2, 87.2, 95.1, 96.3 and 85.8, respectively. Whereas, compounds 5a, 7a, 8b, 10a, 10b, 10c and 10i showed high inhibitions on hRNMT. It is of great value to mention that compounds with high inhibitions toward SARs-Cov nsp 14 containing in most cases the electronegative $\mathrm{Cl}$ group beside the trichlomethyl moiety.

Table 2. IC50 values of the newly synthesized compounds on SARS-CoV nap14 and human RNMT activities

\begin{tabular}{|c|c|c|c|c|c|c|}
\hline Compd & $\mathrm{R}$ & $\mathrm{X}$ & $\mathrm{Y}$ & $\mathrm{X}$ & SARS-Cov & hRNMT \\
\hline
\end{tabular}




\begin{tabular}{|c|c|c|c|c|c|c|}
\hline & & & & & $\begin{array}{c}\text { nap14 } \mathrm{IC}_{50^{\mathrm{a}}} \\
(\mu \mathrm{M})\end{array}$ & $\mathrm{IC}_{50}(\mu \mathrm{M})$ \\
\hline $5 c$ & $\mathrm{CH}_{3}$ & $\mathrm{CN}$ & - & - & $33.4 \pm 4.6$ & $8.6 \pm 2.1$ \\
\hline $5 d$ & $\mathrm{CH}_{3}$ & COOEt & - & - & $58.1 \pm 6.3$ & $70.4 \pm 6.2$ \\
\hline $7 a$ & $\mathrm{H}$ & $\mathrm{CN}$ & - & - & $1.4 \pm 0.89$ & $88.2 \pm 8.3$ \\
\hline $7 b$ & $\mathrm{H}$ & COOEt & - & & $28.8 \pm 4.1$ & $75.6 \pm 4.1$ \\
\hline $7 c$ & $\mathrm{CH}_{3}$ & $\mathrm{CN}$ & - & - & $10.3 \pm 2.6$ & $90.2 \pm 6.4$ \\
\hline $7 d$ & $\mathrm{CH}_{3}$ & COOEt & - & - & $21.6 \pm 4.3$ & $38.6 \pm 2.1$ \\
\hline $8 a$ & $\mathrm{H}$ & $\mathrm{CN}$ & - & - & $0.84 \pm 0.5$ & $68.3 \pm 5.8$ \\
\hline $8 b$ & $\mathrm{H}$ & COOEt & - & - & $46.1 \pm 3.7$ & $72.1 \pm 4.6$ \\
\hline $8 c$ & $\mathrm{CH}_{3}$ & $\mathrm{CN}$ & - & - & $12.4 \pm 2.5$ & $68.2 \pm 3.2$ \\
\hline $8 d$ & $\mathrm{CH}_{3}$ & COOEt & - & - & $8.1 \pm 2.3$ & $77.1 \pm 4.6$ \\
\hline $10 \mathbf{a}$ & $\mathrm{H}$ & $\mathrm{CN}$ & $\mathrm{H}$ & $\mathrm{CN}$ & $1.3 \pm 0.6$ & $<0.05$ \\
\hline $10 \mathrm{~b}$ & $\mathrm{H}$ & $\mathrm{CN}$ & $\mathrm{H}$ & COOEt & $2.6 \pm 0.8$ & $86.3 \pm 5.9$ \\
\hline $10 \mathrm{c}$ & $\mathrm{CH}_{3}$ & $\mathrm{CN}$ & $\mathrm{H}$ & $\mathrm{CN}$ & $0.86 \pm 0.3$ & $94.1 \pm 3.6$ \\
\hline 10d & $\mathrm{CH}_{3}$ & $\mathrm{CN}$ & $\mathrm{H}$ & COOEt & $0.49 \pm 0.1$ & $98.5 \pm 6.2$ \\
\hline $10 \mathrm{e}$ & $\mathrm{H}$ & $\mathrm{CN}$ & $\mathrm{Cl}$ & $\mathrm{CN}$ & $0.52 \pm 0.2$ & $90.2 \pm 8.1$ \\
\hline $10 \mathrm{f}$ & $\mathrm{H}$ & $\mathrm{CN}$ & $\mathrm{Cl}$ & COOEt & $0.34 \pm 0.18$ & $<0.05$ \\
\hline $10 \mathrm{~g}$ & $\mathrm{CH}_{3}$ & $\mathrm{CN}$ & $\mathrm{Cl}$ & $\mathrm{CN}$ & $1.35 \pm 0.81$ & $<0.05$ \\
\hline $10 \mathrm{~h}$ & $\mathrm{CH}_{3}$ & $\mathrm{CN}$ & $\mathrm{Cl}$ & COOEt & $2.3 \pm 0.68$ & $<0.05$ \\
\hline $10 \mathrm{i}$ & $\mathrm{H}$ & $\mathrm{CN}$ & $\mathrm{OCH}_{3}$ & $\mathrm{CN}$ & $10.4 \pm 1.6$ & $40.6 \pm 3.2$ \\
\hline $10 \mathrm{k}$ & $\mathrm{H}$ & $\mathrm{CN}$ & $\mathrm{OCH}_{3}$ & COOEt & $16.5 \pm 2.5$ & $38.3 \pm 4.6$ \\
\hline 101 & $\mathrm{CH}_{3}$ & $\mathrm{CN}$ & $\mathrm{OCH}_{3}$ & $\mathrm{CN}$ & $14.3 \pm 3.1$ & $25.6 \pm 2.9$ \\
\hline $10 \mathrm{~m}$ & $\mathrm{CH}_{3}$ & $\mathrm{CN}$ & $\mathrm{OCH}_{3}$ & COOEt & $19.5 \pm 2.6$ & $42.3 \pm 3.3$ \\
\hline Sinefungin $^{\mathrm{b}}$ & - & - & - & - & 0.36 & $<0.05$ \\
\hline
\end{tabular}

${ }^{a}$ Concentration inhibiting MTase activity by $50 \%$; mean value from three independent experiments. 
It is clear from Table 2 that most of the tested compounds showed IC50's indicated that they are active toward Cov nap14. Compounds 7a, 8a, 10a, 10c, 10d, 10e, 10f and 10g showed IC 50 's $1.4,0.84,1.3,0.86,0.49,0.52,0.34$ and $1.34 \mu \mathrm{M}$. In addition most of the tested compounds were not active toward hRNMT. Interestingly some compounds like 10a, 10f, 10g and 10h showed IC50's $<0.05$ against hRNMT.

\subsection{Materials and Methods \\ Structure retrieval and preparation:}

Subsequently, SCIGRESS3.2 software was used to optimize the molecules using the implemented molecular mechanics force field (MM3) and the semiempirical parameterization method 6 (PM6) [36,37]The optimized structures are checked for any transition state by calculating the Infrared (IR) spectra [38]. The optimized structures were prepared for the docking experiment using the AutoDock Tools software [39] and the AutoDockVina software was utilized in the docking of the halogenated compounds into the active site of SARS-CoV-2M $\mathrm{Mro}^{\text {pro }}$ 40]. The best resolution structure for SARS$\mathrm{CoV}-2 \mathrm{M}^{\mathrm{pro}}$ was used in this study (PDB ID: 6Y84), and it was subjected to equilibration and Molecular Dynamics Simulation (MDS) run for up to 100 ns using Nanoscale Molecular Dynamics (NAMD) software[41]. Following the MDS, the SARS-CoV-2 $\mathrm{M}^{\text {pro }}$ trajectories were clustered using Chimera software into five different clusters [42] and a representative structure from each cluster was used in the docking study.

\section{Molecular Docking study:}

The AutoDockVina software was used to test 22 different halogen-containing compounds against SARS-CoV-2 $\mathrm{M}^{\text {pro }}$, where Nelfinavir was used as a positive control to scale the halogenated compounds' affinity to SARS-CoV-2 $\mathrm{M}^{\text {pro }}$. The Grid box size used in the 
docking experiments was $40 \times 40 \times 40 \AA^{3}$, while the box centerswereset to be at the active site residues $\mathrm{H} 41$ and $\mathrm{C} 145(-3.9,19.7,-5.0) \AA,(-4.6,18.9,-15.5) \AA,(-8.2,37.0,-$ 8.4) $\AA,(4.7,38.8,-3.3) \AA,(22.1,32.0,-2.4) \AA$, for the different conformations of the SARS-CoV-2 $\mathrm{M}^{\text {pro }}$. Following docking, the complexes were investigated using both PyMOL and the Protein-Ligand Interaction Profiler (PLIP) software [43,44].

\section{Results and Discussion}

The effective treatment options for SARS-CoV-2 can either be the use of broad-spectrum antiviral drugs or specific therapeutic molecules thatcan disrupt the viral lifecycle. Since the rapid transmission of coronavirus has proven to be devastating worldwide, several preventive approaches have been suggested by the health care authorities. The implementation of quarantine for infected patients, the monitoring and timely diagnosis of suspected cases, the use of protective masks, andthorough hand washing can help to control disease dissemination [45]. Unfortunately,infection and mortality rates are rising, and no specific COVID-19 treatment has been confirmed to be successful.Thus, extensive research aimed at discovering and developing COVID-19 treatment drugs is urgently warranted.Studies have investigated the therapeutic targeting of the main protease of SARS-CoV-2 $\left(\mathrm{M}^{\mathrm{pro}}\right)$ to combat COVID-19 [46]. In this study, the halogenated compounds exhibit the potential to inhibit the $\mathrm{M}^{\text {pro }}$, albeit without guaranteed activity. Nevertheless, these insightful findings provide a foundation for computational drug discovery of novel compounds to combat SARS-CoV-2.

Generally, halogen bonding interactions in biological systems have been widely acknowledged [47-61]. [Not only did halogen-containing compounds attract attention 
through a fundamental research perspective, but also through drug evolution to meet clinical endpoints [62]. This is exemplified by the formulation of a series of halogencontaining inhibitors of cathepsin after the discovery of hydrogen bonding between selected ligands and its active site [57]. In addition, $\mathrm{Xu}$ et al studied the interaction between sildenafil and its receptor, PDE5, with the goal of improving the binding efficiency between its successors and the receptor [59]. Following the analysis of the atomic details ofreceptor-drug contacts, a molecular docking program was used to replace hydrogen atoms in the drug with halogen atoms that, theoretically, could produce halogen bonds. By utilizing a hybrid method including both quantum and molecular mechanics, $\mathrm{Xu}$ et al were capable of refining the search for successors which were subsequently synthesized [59,62]. The progeny's binding energies with the receptor have been discovered to be in remarkable accordance with the computational predictions. This proves thathalogen bonding could be rationally exploited to impact drug discovery [62].

Table 2shows the average binding energy $(\mathrm{kcal} / \mathrm{mol})$ of the different halogenated compounds against SARS-CoV-2 $\mathrm{M}^{\text {pro }}$, compared to a positive control, Nelfinavir (-6.92 $\pm 0.5 \mathrm{kcal} / \mathrm{mol}$ ). As shown in figure 1 , fifteen of the tested compounds show promising results in terms of binding to the SARS-CoV-2 $\mathrm{M}^{\text {pro }}$ active site. The best three compounds are colored in dark green (compounds 13, 17, and 21) with average binding affinities of $-6.94 \pm 0.36,-7.0 \pm 0.59$, and $-6.98 \pm 0.61$, respectively. Additionally, twelve compounds (light green columns) show comparable results to theNelfinavir (red column), namely compounds $7 \mathrm{a}, 7 \mathrm{c}, 7 \mathrm{~d}, 10 \mathrm{a}, 10 \mathrm{~b}, 10 \mathrm{~d}, 10 \mathrm{e}, 10 \mathrm{f}, 10 \mathrm{~h}, 10 \mathrm{i}, 10 \mathrm{k}$ and $10 \mathrm{~m}$. Conversely, compounds 3a, 3b, 5a, 5b, 5c, 5d and7b (blue columns) show higher 
average binding energies, with less affinity, compared to Nelfinavir against SARS-CoV-2 $\mathrm{M}^{\text {pro }}(-5.92 \pm 0.34$ up to $-4.76 \pm 0.17 \mathrm{kcal} / \mathrm{mol})$.

Table 2. The interaction pattern of the halogenated compounds with better binding affinities than Nelfinavir against SARS-CoV-2 Mro.

\begin{tabular}{|c|c|c|c|c|c|}
\hline \multirow[b]{2}{*}{ Compound } & \multirow{2}{*}{$\begin{array}{c}\text { AutoDockVina } \\
\text { score } \\
\text { (kcal/mol) }\end{array}$} & \multicolumn{2}{|c|}{ H-bonding / Halogen bonding } & \multicolumn{2}{|c|}{ Hydrophobic interactions } \\
\hline & & Number & $\begin{array}{l}\text { Residues from SARS- } \\
\text { CoV-2 } \mathrm{M}^{\text {pro }}\end{array}$ & Number & $\begin{array}{l}\text { Residues from SARS- } \\
\text { CoV-2 } \mathrm{M}^{\text {pro }}\end{array}$ \\
\hline Nelfinavir & -6.7 & 1 & E166 & 3 & T25, L27, and M165 \\
\hline $7 \mathbf{a}$ & -6.3 & 1 & N142 & 1 & Q189 \\
\hline $7 \mathrm{c}$ & -6.2 & 3 & Q189, T190, and A191 & 3 & F181, and V186(2) \\
\hline $7 d$ & -6.3 & 1 & Q189 & 3 & T25, L27, and N142 \\
\hline $10 \mathrm{a}$ & -6.9 & 4 & T24, S46(2), and D187 & 1 & T25 \\
\hline $10 \mathrm{~b}$ & -6.1 & 2 & Q189(2) & 1 & $\mathbf{T 2 5}$ \\
\hline $10 \mathrm{c}$ & -6.9 & 4 & $\begin{array}{c}\mathrm{T} 24, \mathrm{C} 44, \mathrm{~S} 46, \text { and } \\
\text { D187 }\end{array}$ & 1 & T25 \\
\hline 10d & -6.9 & $\begin{array}{l}5 \\
1\end{array}$ & $\begin{array}{c}\text { N142, D187, and } \\
\text { Q189(3), } \\
\text { E47 }\end{array}$ & 2 & T25 and Q189 \\
\hline $10 \mathrm{e}$ & -6.8 & $\begin{array}{l}5 \\
2\end{array}$ & $\begin{array}{c}\text { S46, N142, E166(2), } \\
\text { and Q189 } \\
\text { E166, and T190 }\end{array}$ & 1 & L27 \\
\hline $10 f$ & -6.7 & 3 & T45, S46, and Q189 & 1 & Q189 \\
\hline $10 \mathrm{~g}$ & -6.9 & $\begin{array}{l}1 \\
1\end{array}$ & $\begin{array}{l}\mathrm{S} 144 \\
\mathrm{~T} 25\end{array}$ & 1 & E166 \\
\hline $10 \mathrm{~h}$ & -6.3 & $\begin{array}{l}2 \\
2\end{array}$ & $\begin{array}{l}\text { T24 and T45 } \\
\text { E47 and E166 }\end{array}$ & 1 & Q189 \\
\hline $10 \mathrm{i}$ & -7.0 & $\begin{array}{l}1 \\
1\end{array}$ & $\begin{array}{l}\text { N142 } \\
\text { N142 }\end{array}$ & 1 & T25 \\
\hline $10 \mathrm{k}$ & -6.8 & 5 & $\begin{array}{l}\text { N142, G143, S144, } \\
\text { C145, and D187 }\end{array}$ & 2 & M165 and E166 \\
\hline 101 & -7.0 & 4 & $\begin{array}{c}\text { T26, S46, E166, and } \\
\text { Q189 }\end{array}$ & 1 & N142 \\
\hline $10 \mathrm{~m}$ & -6.5 & $\begin{array}{l}4 \\
1\end{array}$ & $\begin{array}{c}\mathrm{T} 24, \mathbf{T} 25(2), \text { and } \mathbf{E} 166 \\
\mathrm{E} 47\end{array}$ & 0 & \\
\hline
\end{tabular}

The AutoDockVina scores are listed among the number of H-bonds and hydrophobic contacts and the residues that interact. The green residue is the one that interacted with halogen bonds with the ligands.

Bold residues are the most common residues. 


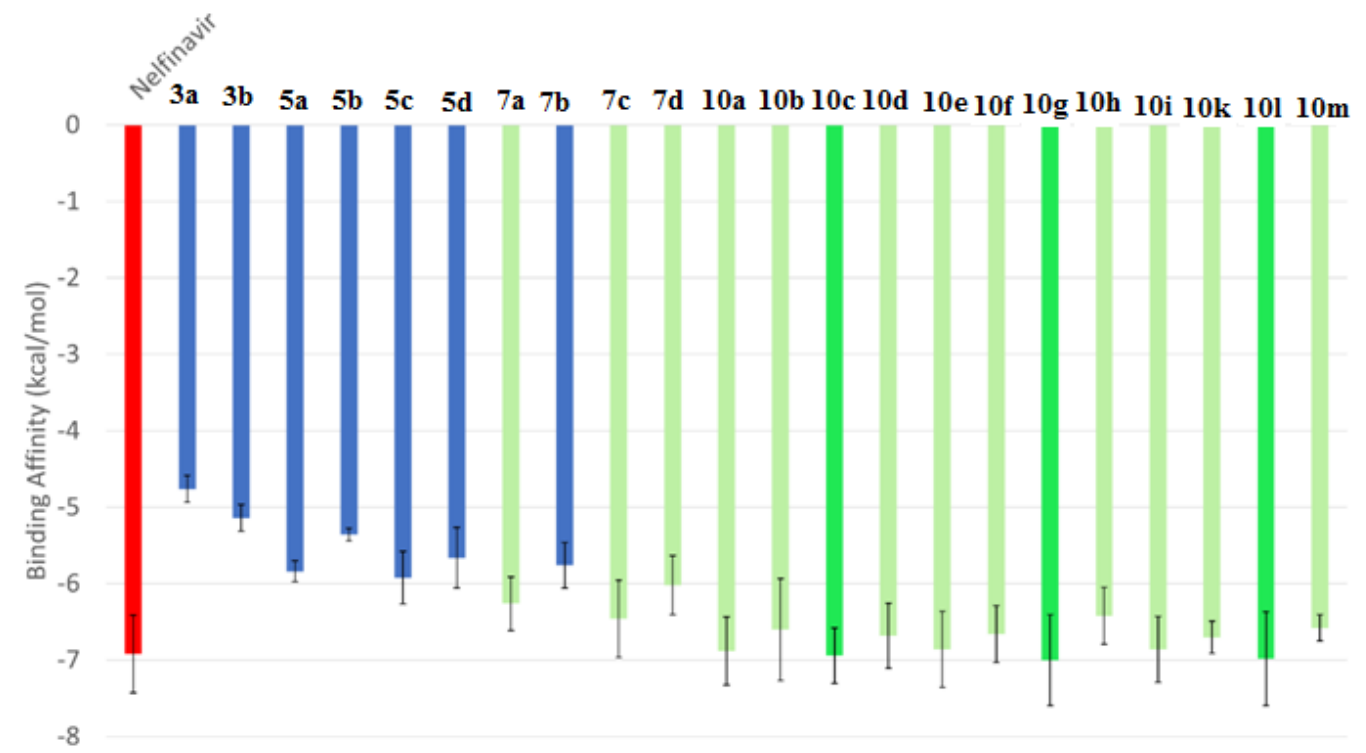

Figure 1: The average binding energies (kcal/mol) of 22 halogenated compounds and Nelfinavir against SARS-CoV-2 Mpro active site residue H41 and C145. Error bars represent the standard deviation (SD). The halogenated compounds are classified into top compounds (dark green), compounds with comparable binding energywithNelfinavir (light green), and compounds that bind SARS-CoV-2 Mpro with less affinity compared with Nelfinavir (blue), while Nelfinavir is red-colored.

A representative docking complex was selected for further analysis of each compound. Table 2 shows the PLIP analysis of the compounds after docking when the main interactions established were Hydrogen, halogen bonds and hydrophobic contacts. It is noteworthy that the most common residues that interactedwith the halogenated compounds were T25, N142, E166, D187 and Q189.The best three compounds in terms of binding affinity (compounds 13,17, and 21) are depicted in figure 2 with stick representation. As reported from both Table 2 and Figure 2, the most common residues that contributed to the interaction with the halogenated compounds wereT25, E166, D187, and Q189. 


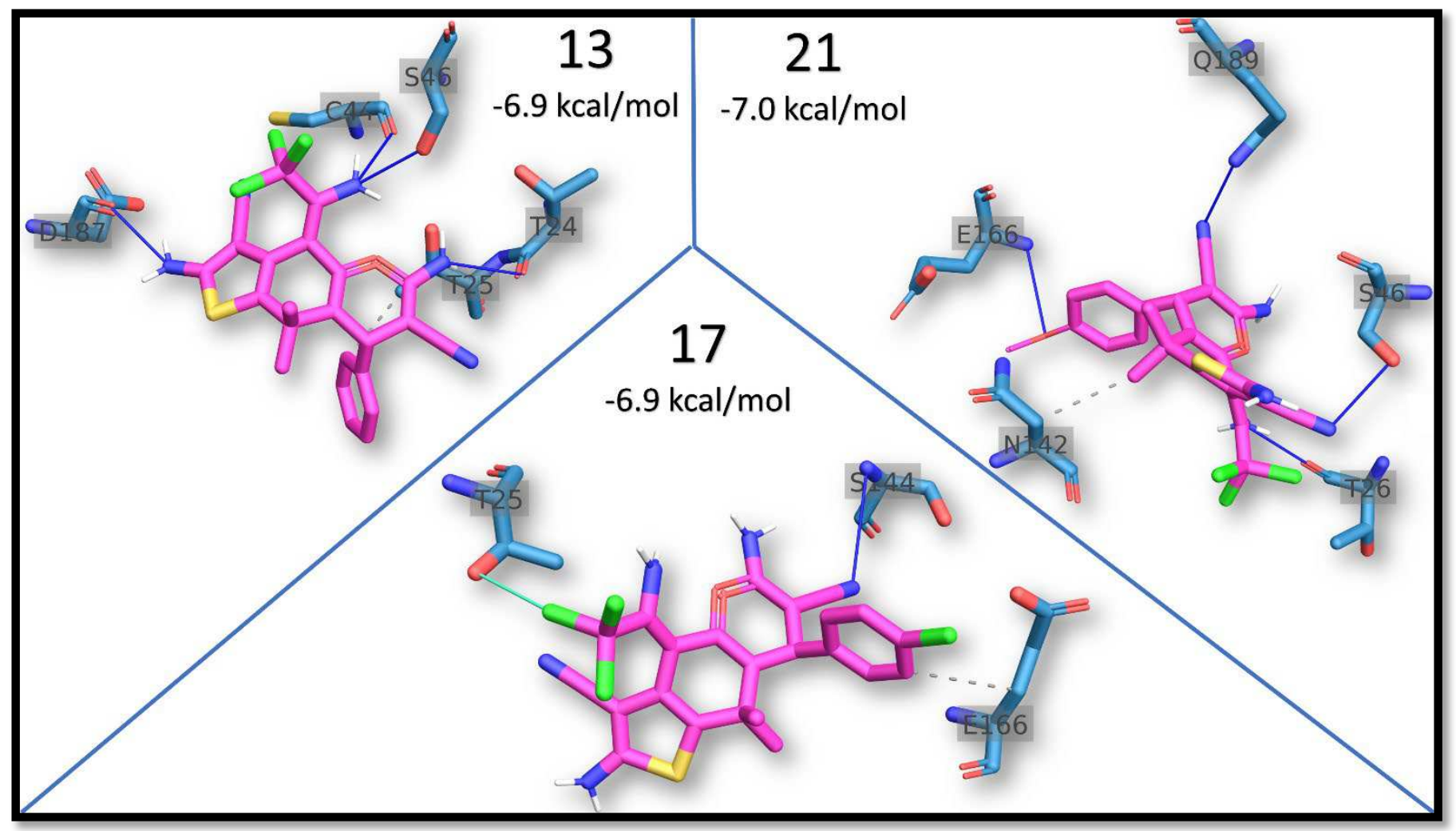

Figure 2: The interaction pattern for the docking of the best three halogenated compounds (13, 17, and 21) against the active site of SARS-CoV-2 $\mathrm{M}^{\text {pro }}$. H-bonds and the hydrophobic contacts are represented in blue and dashed-gay lines, respectively.Halogen bonds are depicted in green lines. The interacting residues are labeled with their oneletter codes, while the colored sticks represent both the halogenated compounds (magenta) and the interacting residues from SARS-CoV-2 $\mathrm{M}^{\text {pro }}$ (blue).

Noticeably, the residues E166 and M165 were involved in the interaction between Nelfinavir and the SARS-CoV-2 $\mathrm{M}^{\text {pro }}$. The former formsan $\mathrm{H}$-bond with an $\mathrm{OH}$ group located in the meta-position of the benzyl ring, while the latter forms a hydrophobic contact with a methyl group in the orthoposition of the same benzyl ring. The superposition of the docking complexes from Nelfinavir and the compounds 13, 17, and 21 is shown in figure 3. As reflected from the figure, thethree halogenated compounds reside in the active site pocket of the SARS-CoV-2 $\mathrm{M}^{\text {pro }}$, superimposed over Nelfinavir suggesting the same mode of interaction, hence possible inhibition of the main protease 
of SARS-CoV-2. These results are in support of previous studies on the effectiveness of

halogen-containing compounds as potential antiviral agents $[63,64]$.

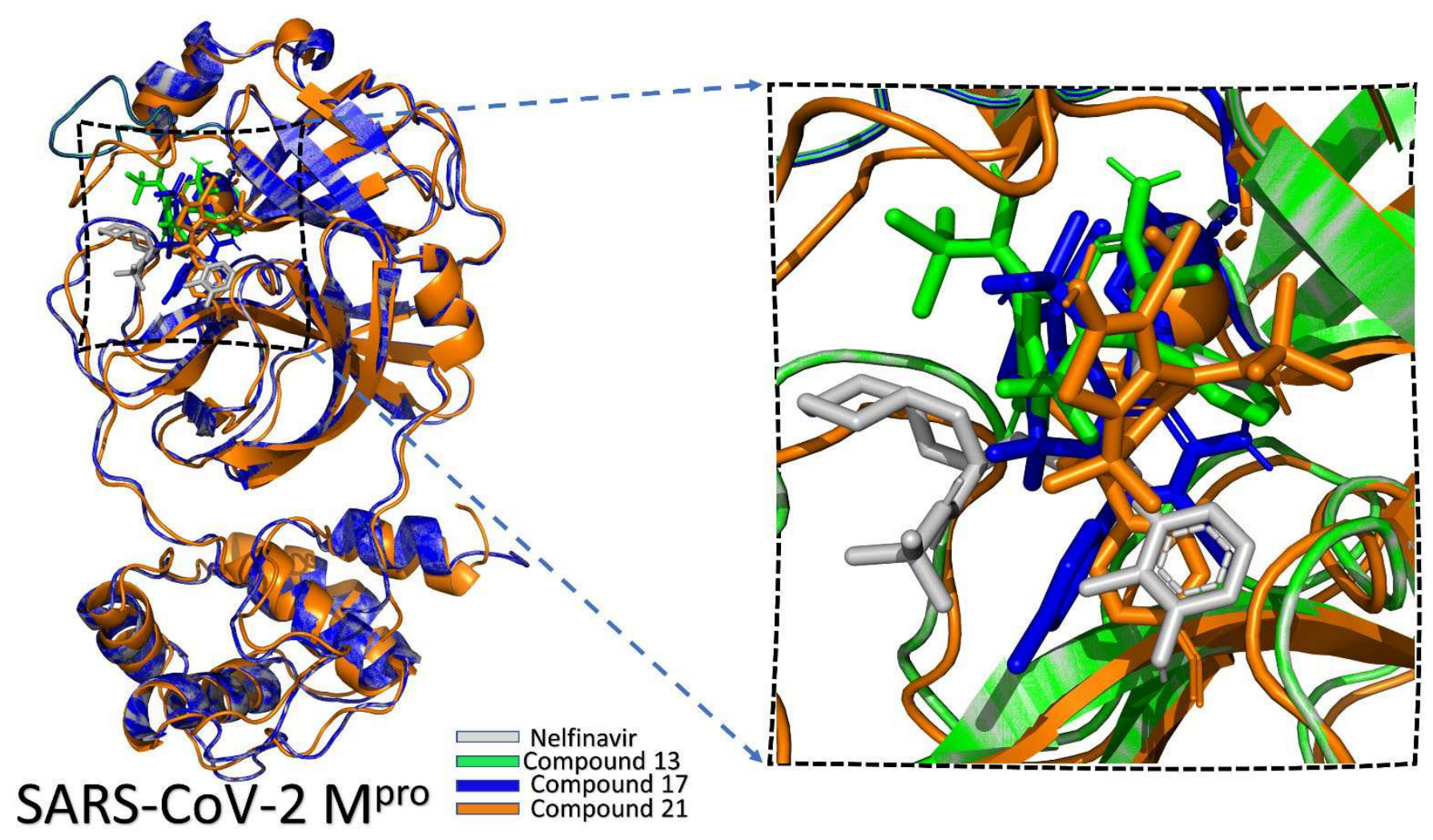

Figure 3 The superposition of the docking complexes of Nelfinavir (gray), and the compounds $\mathbf{1 0 c}$ (green), $\mathbf{1 0 g}$ (blue), and $\mathbf{1 0 l}$ (orange) docked into the active site of SARS$\mathrm{CoV}-2 \mathrm{M}^{\text {pro }}$. The enlarged panel is depicted to show how the compounds are lying in the active site cavity of the protein.

\section{Results and discussion}

The reaction of either cyclohexan-1,3-dione (1a) or dimedone (1b) with trichloroacetonitrile (2) gave the 2,2,2-trichloroethylidene)cyclohexane-1,3-dione derivatives $\mathbf{3} \mathbf{a}$ and $\mathbf{3 b}$, respectively. Structures of the latter products were confirmed of the basis of their respective analytical and spectral data (see experimental section). Compounds 3a and $\mathbf{3 b}$ were capable for thiophene synthesis through the well known Gewals's thiophene synthesis [65-67]. Thus, the reaction of either 3a or $\mathbf{3 b}$ with each of elemental sulfur and either malononitrile (4a) or ethyl cyanoacetate (4b) gave the 2,2,2trichloroethylidene)-6,7-dihydrobenzo[b]thiophen-5(4H)-one5a and $\mathbf{5 b}$, respectively. The analytical and spectral data of compounds 5a-d were in agreement of their strutuctures. 
Thus, the ${ }^{1} \mathrm{H}$ NMR spectrum of $\mathbf{5 a}$ (as an example) showed the presence of two singlets at $\delta 5.25$ and $4.80 \mathrm{ppm}\left(\mathrm{D}_{2} \mathrm{O}\right.$ exchangeable) for the two $\mathrm{NH}_{2}$ groups and two triplets at $\delta$ 2.13 and $2.45 \mathrm{ppm}$ equivalent for the two $\mathrm{CH}_{2}$ group. In addition, the ${ }^{13} \mathrm{C} \mathrm{NMR}$ spectrum revealed the presence of a signal at $\delta 168.2$ equivalent to the $\mathrm{C}=\mathrm{O}$ group, four signals at $\delta 132.6,133.8,138.0,140.1$ indicating the four thiophene carbons, a signal at $\delta$ 116.8 for the $\mathrm{CN}$ group, two signals at $\delta 112.1$ and 90.6 for the ethylidene carbons, a signal at $\delta 94.4$ for the $\mathrm{CCl}_{3}$ group and two signals at $\delta 40.7$ and 39.6 for the two $\mathrm{CH}_{2}$ groups. The reaction of either 5a-d with phenylisothiocyanate (6) gave the 4,6dihydrothieno[3,2-f]quinazoline-3-thiol derivatives 7a-d, respectively (Scheme 1). The analytical and spectral data of the latter compounds were in agreement with their structures. 

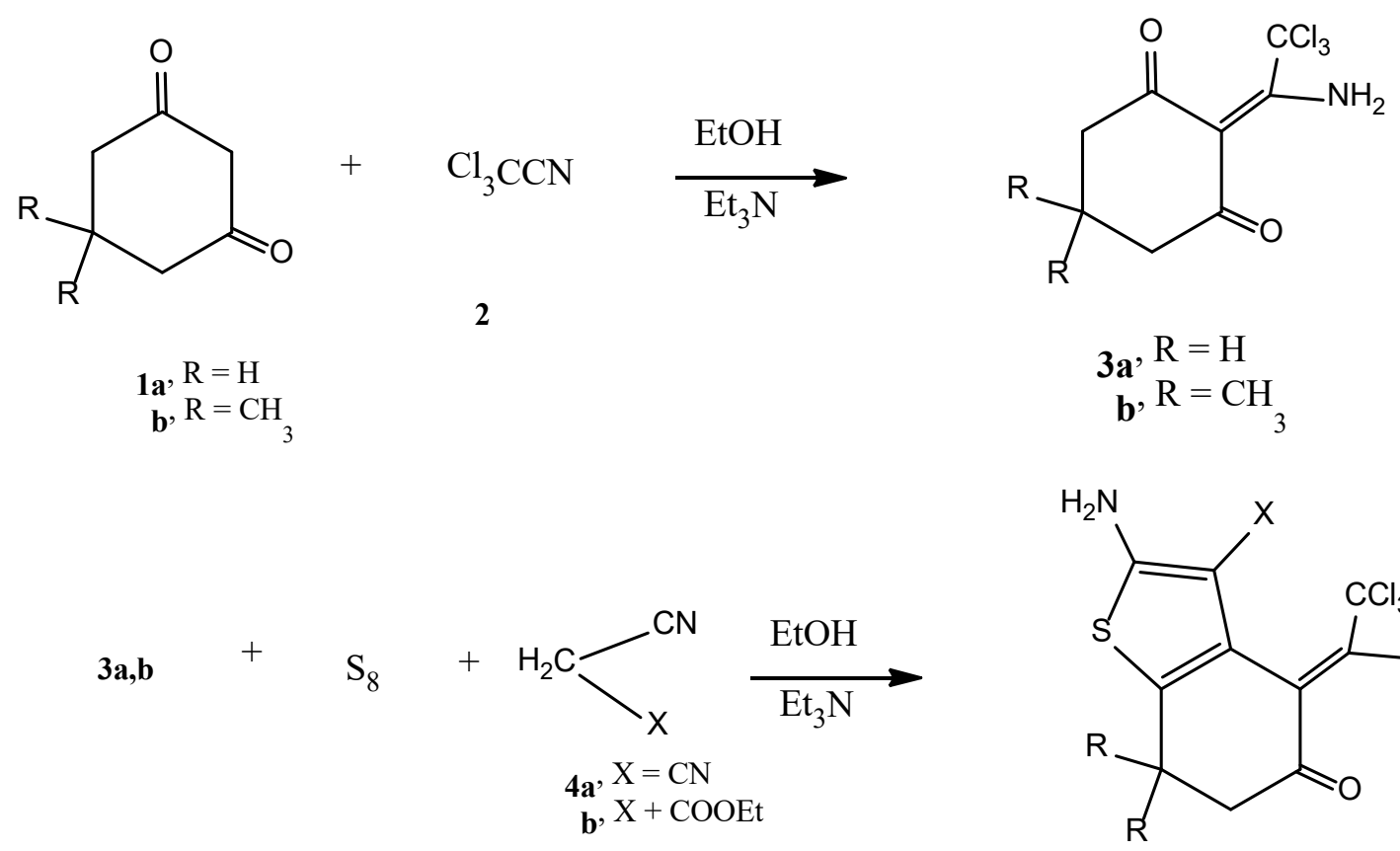

$$
\begin{aligned}
\mathbf{3 a}, \mathrm{R} & =\mathrm{H} \\
\mathbf{b}, \mathrm{R} & =\mathrm{CH}_{3}
\end{aligned}
$$

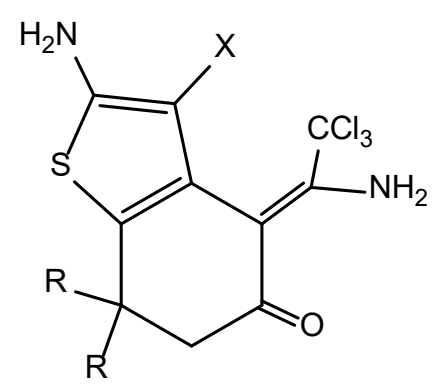

$$
\begin{aligned}
\text { 5a } & R=H, X=C N \\
\text { b } & R=H, X=C O O E t \\
\text { c, } & R=C H, X=C N \\
\text { d, } & R=\mathrm{CH}_{3}^{3}, X=C O O E t
\end{aligned}
$$
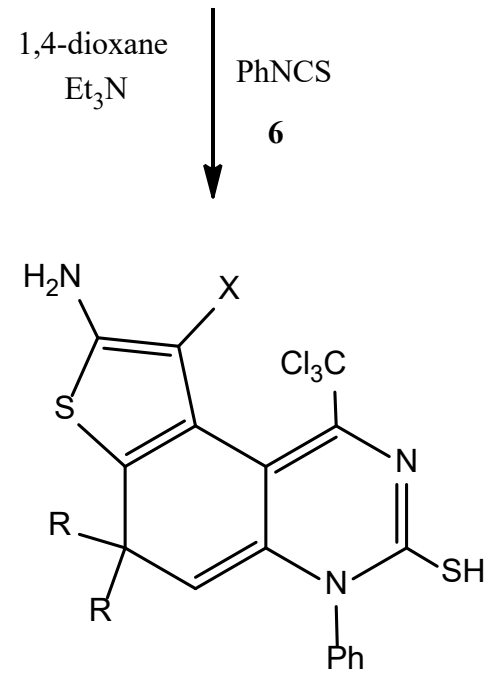

$$
\begin{aligned}
& \text { 7a, } \mathrm{R}=\mathrm{H}, \mathrm{X}=\mathrm{CN} \\
& \text { b, } \mathrm{R}=\mathrm{H}, \mathrm{X}=\mathrm{COOEt} \\
& \text { c, } \mathrm{R}=\mathrm{CH}, \mathrm{X}=\mathrm{CN} \\
& \text { d, } \mathrm{R}=\mathrm{CH}_{3}^{3}, \mathrm{X}=\mathrm{COOEt}
\end{aligned}
$$

Scheme 1. Synthesis of compounds $\mathbf{3 a , b} ; \mathbf{5 a - d}{ }^{\text {and }} \mathbf{7 a - d}$ 
Compounds 7a-d underwent ready hydrolysis of the trichloromethylmoiety to give the 4,6-dihydrothieno[3,2-f]quinazolin-1-ol derivatives 8a-d, respectively (Scheme 2).<smiles>[X]c1c(N)sc2c1C1=C(C)N=C(S)N(c3ccccc3)C1=CC2([R])[2H]</smiles>

$$
\begin{aligned}
7 \mathbf{a}, & \mathrm{R}=\mathrm{H}, \mathrm{X}=\mathrm{CN} \\
\mathbf{b}, \mathrm{R} & =\mathrm{H}, \mathrm{X}=\mathrm{COOEt} \\
\mathbf{c}, \mathrm{R} & =\mathrm{CH}, \mathrm{X}=\mathrm{CN} \\
\mathbf{d}, \mathrm{R} & =\mathrm{CH}_{3}^{3}, \mathrm{X}=\mathrm{COOEt}
\end{aligned}
$$

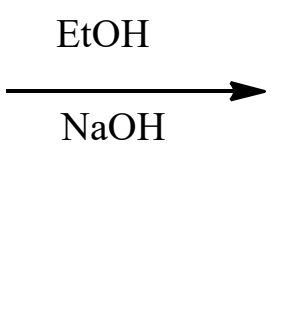

Scheme 2 . Synthesis of compounds $\mathbf{8 a - d}$.

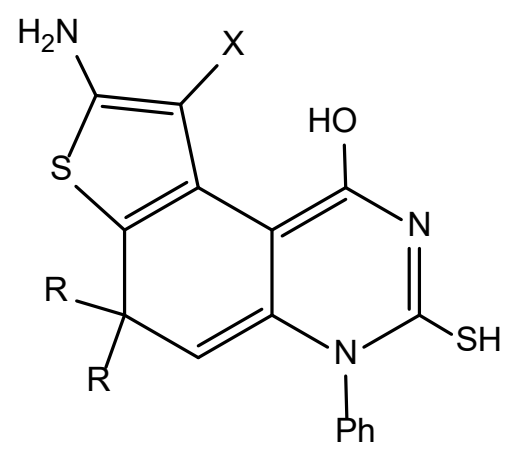

$$
\begin{aligned}
\mathbf{8 a}^{2}, \mathrm{R} & =\mathrm{H}, \mathrm{X}=\mathrm{CN} \\
\mathbf{b}, \mathrm{R} & =\mathrm{H}, \mathrm{X}=\mathrm{COOEt} \\
\mathbf{c}, \mathrm{R} & =\mathrm{CH}, \mathrm{X}=\mathrm{CN} \\
\text { d }, \mathrm{R} & =\mathrm{CH}_{3}^{3}, \mathrm{X}=\mathrm{COOEt}
\end{aligned}
$$

In recent years, multicomponent reactions (MCRs) have become essential, efficient, bond-forming methods for expedient synthesis of a wide range of active organic compounds and natural products without separation and purification of intermediates. The MCRs, which are important classes of chemical transformations, have recently attracted much attention owing to their high efficacy, shorter reaction times, mild conditions, simplicity, and environmental friendliness [68-71]. During the last several years, the diverse applications of such2-amino- $4 H$-pyran heterocyclic scaffolds in medicinal chemistry have drawn appreciable attention among synthetic chemiststo 
explore useful synthetic routes to these heterocycles of potentialinterest with antimicrobial and antitumor activities [72,73]. Compounds 5a,c were ready for multicomponent reactions due to the presence of $\alpha$-oxomethlene moiety within such molecules. Therefore, the reaction of either of $5 \mathbf{a}$ or $\mathbf{5} \mathbf{c}$ with either benzaldehyde (9a), 4chlorobenzaldehyde (9b) or 4-methoxybenzaldehyde (9c) and either malononitrile (4a) or ethyl cyanoacetate $(\mathbf{4 b})$ in 1,4-dioxane containing a catalytic amount of triethylamine gave the 8,9-dihydro- $4 H$-thieno[2,3-g]chromene derivatives 10a-m, respectively (Scheme 3). The analytical and spectral data of the latter compounds were in agreement with their respective structures. Thus, the ${ }^{1} \mathrm{H}$ NMR spectrum of compound 10a (as an example) revealed the presence of a multiplet at $\delta 7.25-7.42 \mathrm{ppm}$ for the phenyl protons, a singlet at $\delta 5.90$ equivalent to the pyran $H-4$, three signals at $\delta 4.80,5.22,5.39 \mathrm{ppm}$ ( $\mathrm{D}_{2} \mathrm{O}$ exchangeable) for the three $\mathrm{NH}_{2}$ groups and a singlet at $\delta 2.80 \mathrm{ppm}$ for the $\mathrm{CH}_{2}$ group. In addition the ${ }^{13} \mathrm{C}$ NMR spectrum which showed singles at $\delta 120.1,123.5$, $125.4,128.5,130.8,133.7,137.9,138.3,139.0,140.2,142.5,143.8$ equivalent to the $\mathrm{C}_{6} \mathrm{H}_{5}$, thiophene, pyran carbons, two signals at $\delta 116.9,117.3$ for the two $\mathrm{CN}$ groups, a signal at $\delta 94.2$ for the $\mathrm{CCl}_{3}$ group and a signal at $\delta 53.8$ corresponding to the $\mathrm{CH}_{2}$ group. 

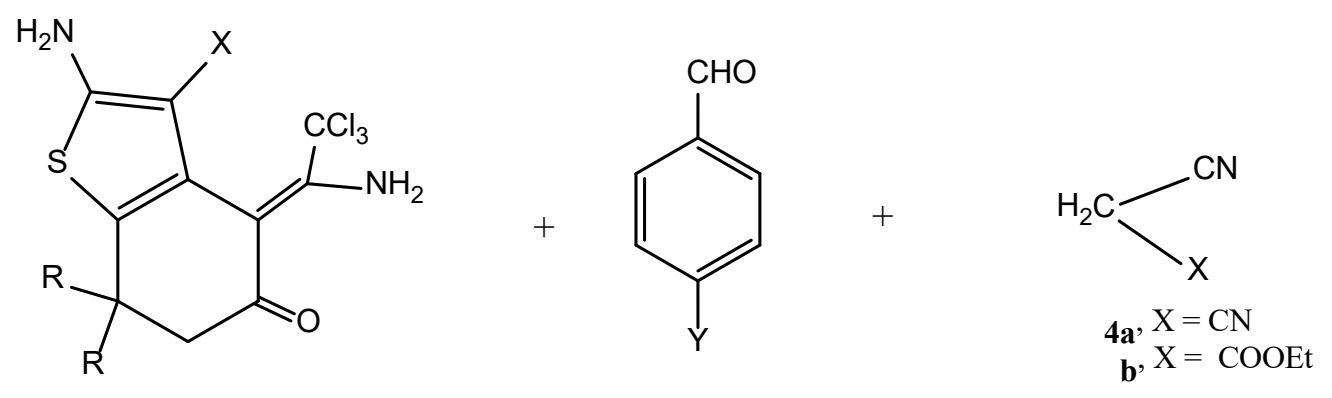

$$
\begin{aligned}
\mathbf{5 a}, \mathrm{R} & =\mathrm{H}, \mathrm{X}=\mathrm{CN} \\
\text { c, } \mathrm{R} & =\mathrm{CH}_{3}, \mathrm{X}=\mathrm{CN}
\end{aligned}
$$$$
\begin{aligned}
\mathbf{9 a}, Y & =\mathrm{H} \\
\text { b, }, & =\mathrm{Cl} \\
\text { c, } Y & =\mathrm{OCH}_{3}
\end{aligned}
$$

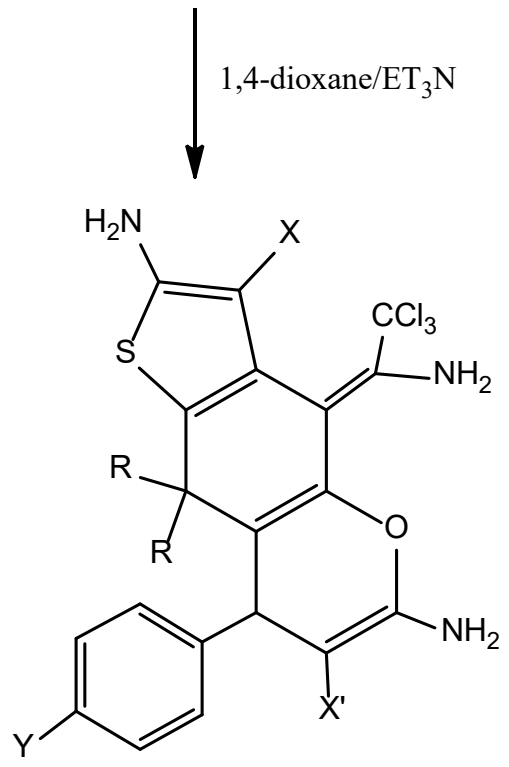

\begin{tabular}{|c|c|c|c|c|c|c|c|c|c|c|c|c|}
\hline $\mathbf{1 0}$ & $\mathbf{a}$ & $\mathbf{b}$ & $\mathbf{c}$ & $\mathbf{d}$ & $\mathbf{e}$ & $\mathbf{f}$ & $\mathbf{g}$ & $\mathbf{h}$ & $\mathbf{i}$ & $\mathbf{k}$ & $\mathbf{l}$ & $\mathbf{m}$ \\
\hline $\mathrm{R}$ & $\mathrm{H}$ & $\mathrm{H}$ & $\mathrm{CH}_{3}$ & $\mathrm{CH}_{3}$ & $\mathrm{H}$ & $\mathrm{H}$ & $\mathrm{CH}_{3}$ & $\mathrm{CH}_{3}$ & $\mathrm{H}$ & $\mathrm{H}$ & $\mathrm{CH}_{3}$ & $\mathrm{CH}_{3}$ \\
\hline $\mathrm{X}$ & $\mathrm{CN}$ & $\mathrm{CN}$ & $\mathrm{CN}$ & $\mathrm{CN}$ & $\mathrm{CN}$ & $\mathrm{CN}$ & $\mathrm{CN}$ & $\mathrm{CN}$ & $\mathrm{CN}$ & $\mathrm{CN}$ & $\mathrm{CN}$ & $\mathrm{CN}$ \\
\hline $\mathrm{Y}$ & $\mathrm{H}$ & $\mathrm{H}$ & $\mathrm{H}$ & $\mathrm{H}$ & $\mathrm{Cl}$ & $\mathrm{Cl}$ & $\mathrm{Cl}$ & $\mathrm{Cl}$ & $\mathrm{OCH}_{3}$ & $\mathrm{OCH}_{3}$ & $\mathrm{OCH}_{3}$ & $\mathrm{OCH}_{3}$ \\
\hline $\mathrm{X}^{\prime}$ & $\mathrm{CN}$ & $\mathrm{COOEt}$ & $\mathrm{CN}$ & $\mathrm{COOEt}$ & $\mathrm{CN}$ & $\mathrm{COOEt}$ & $\mathrm{CN}$ & $\mathrm{COOEt}$ & $\mathrm{CN}$ & $\mathrm{COOEt}^{2} \mathrm{CN}$ & $\mathrm{COOEt}$ \\
\hline
\end{tabular}

Scheme 3 . Synthesis of compounds $\mathbf{1 0 a - m}$. 


\section{Conclusion:}

Halogenated compounds have been widely acknowledged in the field of medicinal chemistry owing to their binding potentials. that $10 \mathrm{a}, 10 \mathrm{~b}, 10 \mathrm{c}, 10 \mathrm{e}, 10 \mathrm{f}, 10 \mathrm{~g}$ and $10 \mathrm{~h}$ showed high \% inhibitions against SARs-Cov nsp 14. Whereas, compounds 5a, 7a, 8b, 10a, 10b, 10c and 10i showed high inhibitions on hRNMT.this study explored the binding affinity of 22 halogenated compounds to the SARS-CoV-2 $\mathrm{M}^{\text {Pro }}$ and discovered 15 compounds with higher binding affinity thanNelfinavir, of which 3 showed remarkable results. In times of dire need for anti-COVID-19 treatment, our results lay the foundation for further exploratory research on these candidate compounds to examine their mechanism of action and efficacy against corona virus.

\section{ETHICS APPROVAL AND CONSENT TO PARTICIPATE}

This study was approved by the local Research Ethics Committee at the University of Tabuk, Kingdom of Saudi Arabia.

\section{FUNDING}

This study was funded by a research grant ( 1441- 0190 ) from the University of Tabuk, Kingdom of Saudi Arabia.

\section{CONFLICT OF INTEREST}

The authors declare that there are no conflicts of interest.

\section{References}

1. Abbasia, A.A.; Iqbal, J.; Kiran, F.; Ahmad, R.; Kanwal, S.; Munir, A.; Uddin, S.; Nasi,r J.A.; Chalgham, W.; Mahmood, T. (2020) Green formulation and chemical 
characterizations of Rhamnella gilgitica aqueous leaves extract conjugated NiONPs and their multiple therapeutic properties, J. of Molec. Structure, 1218, 128490.

2. Goldsmith, M.R.; Velez, R.T.; Transue, T.R.; Little, S.B.; Rabinowitz, J.R.; Dary C.C. (2009) Comparative in silico modeling of environmental and therapeutic classes of perfluorinated chemicals (PFCS): ADME properties, virtual receptor profiling and generalized PBPK models, Reproductive Toxicology, 27, 419-420.

3. Schiavon, O.; Caliceti, P.; Ferruti, P.; Veronese, F.M. (2000) Therapeutic proteins: a comparison of chemical and biological properties of uricase conjugated to linear or branched poly(ethylene glycol) and poly(N-acryloylmorpholine), Il Farmaco, 55, 264269.

4. Liang, H.; Hu, B.; Chen. L.; Wang, S.; Aorigelec (2020) Recognizing novel chemicals/drugs for anatomical therapeutic chemical classes with a heat diffusion algorithm, Biochimica et Biophysica Acta (BBA) - Molecular Basis of Disease, 11, 165910.

5. Tosstorff, A.; Menzen, T.; Winter, G. (2020) Exploring Chemical Space for New Substances to Stabilize a Therapeutic Monoclonal Antibody. Journal of Pharmaceutical Sciences, 109, 301-307.

6. Talebi, M.; Talebi, M.; Farkhondeh, T.; Samarghandian, S. (2020) Molecular mechanism-based therapeutic properties of honey. Biomedicine \& Pharmacotherapy 130, 110590 .

7. Marican, A.; Doria, O.F.; Polo, E.; Gallego, J.; Lara, E.F. (2020) Data of preparation and evaluation of supramolecular hydrogel based on cellulose for sustained release of therapeutic substances with antimicrobial and wound healing properties. Data in Brief, 31,105902.

8. Li, D.; Li, Z.; Yang, Y.; Li, X.Y.; Du, X.; Zhu, X.D. (2020) Circular RNAs as biomarkers and therapeutic targets in environmental chemical exposure-related diseases. Environmental Research, 180, 108825.

9. Zhi, X.Y.; Jiang, L.Y.; Li, T.; Song, L.L.; Juan, L.; Cao, W.H.; Yang, C. (2020) Natural product-based semisynthesis and biological evaluation of thiol/amino-Michael adducts of xanthatin derived from Xanthium strumarium as potential pesticidal agents. Bioorganic Chemistry 97, 103696.

10. Lv, M.; Zhang, Y.; Wang, F.; Zhang, S.; Xu, H. (2020) Non-food renewable and bioactive forest products for pest management: Valuation of agricultural properties of podophyllotoxin analogs derived from Podophyllum hexandrum as botanical pesticides. Industrial Crops and Products 153, 112608. 
11. Garrido, I.; Flores, P.; Hellín, P.; Vela, N.; Navarro, S.; Fenolla. J. (2020) Solar reclamation of agro-wastewater polluted with eight pesticides by heterogeneous photocatalysis using a modular facility. A case study. Chemosphere, 249, 126156.

12. Musarurwa, H.; Tavengwa, N.T. (2020) Supramolecular solvent-based microextraction of pesticides in food and environmental samples. Talanta, 26, 121515.

13. Yao, Y.; Xie, Y.; Zhao, B.; Zhou, L.; Shi, Y.; Wang, Y.; Sheng, Y.; Zhao, H.; Sun, J.; Cao, H. (2020) N-dependent ozonation efficiency over nitrogen-containing heterocyclic contaminants: A combined density functional theory study on reaction kinetics and degradation pathways. Chemical Engineering Journal, 382, 122708.

14. Mi, Y.; Zhang, J.; Chen, Y.; Sun, X.; Tan, W.; Li, Q,; Guo, Z. (2020) New synthetic chitosan derivatives bearing benzenoid/heterocyclic moieties with enhanced antioxidant and antifungal activities. Carbohydrate Polymers, 249, 116847.

15. Havasi, M.H.; Ressler, A.J.; Parks, E.L.; Cocolas, A.H.; Weaver, A.; Seeram N.P, Henry G.E. (2020) Antioxidant and tyrosinase docking studies of heterocyclic sulfide derivatives containing a thymol moiety. Inorganica Chimica Acta, 505, 119495.

16. Wang, S.; Bao, L.; Song, D.; Wang, J.; Cao, X. (2019) Heterocyclic lactam derivatives containing piperonyl moiety as potential antifungal agents. Bioorg.\& Med. Chem. Lett., 29, 126661.

17. Zimmermann, L.A.; Moraes, M.H.; Rosa, R.; Melo, E.B.; Paula, F.R. Schenkel EP, Steindel M, Bernardes LSC. (2018) Synthesis and SAR of new isoxazole- triazole bis-heterocyclic compounds as analogues of natural lignans with antiparasitic activity. Bioorg \& Med Chem., 26, 4850-4862.

18. Wang, S.; Bao, L.; Wang, W.; Song, D.; Wang, J.; Cao, X. (2018) Heterocyclic pyrrolizinone and indolizinones derived from natural lactam as potential antifungal agents. Fitoterapia, 129, 257-266.

19. Önal, H.T.; Yuzer, A.; Ince, M.; Ayaz, F. (2020) Photo induced anti-inflammatory activities of a Thiophene substituted subphthalocyanine derivative. Photodiagnosis and Photodynamic Therapy, 30, 101701.

20. Muğlu, H.; Yakan, H.; Shouaib, H.A. (2020) New 1,3,4-thiadiazoles based on thiophene-2-carboxylic acid: Synthesis, characterization, and antimicrobial activities. Journal of Molecular Structure, 1203, 127470.

21. Opsenica, I.; Filipovic, V.; Nuss, J. E.; Gomba, L.M.; Opsenica, D.; James, C.; Burnett, J.C., Gussio, R.; Solaja, B.A.; Bavarif, S. (2012) The synthesis of 2,5-bis(4- 
amidinophenyl)thiophene derivatives providing submicromolar-range inhibition of the botulinum neurotoxin serotype A metalloprotease. Eur. J. Med. Chem., 53, 374-379.

22. Parai, M.K.; Panda, G.; Chaturvedi, V.; Manju, Y.K.; Sinha, S. (2008) Thiophene containing triarylmethanes as antitubercular agents. Bioorg \& Med Chem Lett., 18, 289292.

23. Chekol, W.B.; Melesse, D.Y. (2020) Operating room team safety and perioperative anesthetic management of patients with suspected or confirmed novel corona virus in resource limited settings: A systematic review. Trends in Anaesthesia and Critical Care 2020, 34, 14-22.

24. Michot, J.M.; Albiges, L.; Chaput, N.; Saasa, V.; Pommeret, F.; Griscelli, F.; Balleyguler, C.; Besse, B.; Marabelle, A.; Netzer, F.; Merad, M.; Robert, C.; Barlesi, F.; Gachot, B.; Stoclin, A. (2020) Tocilizumab, an anti-IL-6 receptor antibody, to treat COVID-19-related respiratory failure: a case report. Annals of Oncology, 31, 961964.

25. DiLiu, D.; Wang, Q.; Zhang, H.; Cui, L.; Shen, F.; Chen, Y.; Sun, J.; Gan, J.; Sun, J.; Wang, J.; Zhang, J.; Cai, Q.; Deng, J.; Jiang, J.; Zeng, L. (2020) Viral sepsis is a complication in patients with Novel Corona Virus Disease (COVID-19). Medicine in Drug Discovery, 8, 100057.

26. Pati, S.; Houston, T. (2020) Assessing the risk of seizures with chloroquine or hydroxychloroquine therapy for COVID-19 in persons with epilepsy. Epilepsy Research, $165,106399$.

27. Uzunova, K.; Filipova, E.; Pavlova, V.; Vekov, T. (2020) Insights into antiviral mechanisms of remdesivir, lopinavir/ritonavir and chloroquine/hydroxyl-chloroquine affecting the new SARS-CoV-2. Biomedicine \& Pharmacotherapy 131, 110668.

28. Didehban, K.; Vessally, E.; Salary, M.; Edjlali, L.; Babazadeh, M. (2018) Synthesis of a variety of key medicinal heterocyclic compounds via chemical fixation of $\mathrm{CO}_{2}$ onto o-alkynylaniline derivatives. Journal of $\mathrm{CO}_{2}$ Utilization 23, 42-50.

29. Bolchi, C.; Bavo, F.; Appiani, R.; Roda, G.; Pallavicini, M. (2020) 1,4Benzodioxane, an evergreen, versatile scaffold in medicinal chemistry: A review of its recent applications in drug design. Eur. J. Med. Chem. 200, 112419.

30. Takahashi, H.; Iwasaki Y.; Watanabe, T.; Ichinose, N.; Okada, Y.; Oiwa, A.; Kobayashi, T.; Moriya, M.; Oda, T. (2020) Case studies of SARS-CoV-2 treated with favipiravir among patients in critical or severe condition. International Journal of Infectious Diseases, 100, 283-285. 
31. Kinoshita, K.; Matsumoto, K.; Kurauchi. Y.; Hisatsune, A.; Seki, T.; Katsuki. H. (2019) A Nurr1 agonist amodiaquine attenuates inflammatory events and neurological deficits in a mouse model of intracerebral hemorrhage. J. of Neuroimm, 330, 48-54.

32. Liu, L.; Santi, D.V. (1994) 5-Fluoro-2'-deoxycytidine 5'-monophosphate is a mechanism-based inhibitor of thymidylate synthase.Biochimica et Biophysica Acta (BBA) - Protein. Structure and Molecular Enzymology, 1209, 89-94.

33. Tan, H.; He, L.; Cheng Z. (2020) Inhibition of eIF4E signaling by ribavirin selectively targets lung cancer and angiogenesis. Bioch..and Bioph. Res.Comm., 529, 519-525.

34. Pauwels, R.; Balzaeini, J.; Baba, M.; Snoeck, R.; Schols, D.; Herdewijn, P.; Desmyter, J.; De Clercq, E.(1988) Rapid and automated tetrazolium-based colorimetric assay for the detection of anti-HIV compounds. J. Vitrol Methods, 20, 309-321.

35. Peyane, F.; Selisko, B.; Decroly, E.; Vasseur, J.J.; Benarroach, D.; Canard, B.; Alvarez, K. (2007) High-yield production of short GpppA- and 7meGppA-capped RNAs and HPLC-monitoring of methyltransfer reactions at the guanine-N7 and adenosine-2'O position. Nucleic Acids Res., 35, e26.

36. Bikadi, Z.; Hazai, E. (2009) Application of the PM6 semi-empirical method to modeling proteins enhances docking accuracy of Auto Dock. J. Cheminform, 1, 15.

37. Lii, J.H.; Allinger, N.L. (1989) Molecular mechanics. The MM3 force field for hydrocarbons. 3. The van der Waals' potentials and crystal data for aliphatic and aromatic hydrocarbons. $J$. of the Am. Chem. Soc. 111, 8576-8582.

38. Summers, K.L., Anjan P.K., Mahrok M.D.M.; Martin, J.S. (2012) Structural properties of metal-free apometallothioneins. Biochem. Biophys. Res. Commun. 425, 485492.

39. Morris, G.M.; Huey, R., Lindstrom, W.; Sanner, M.F.; Belew, R.K.; Goodsell, D.S.; Olson, A.J. (2009) AutoDock4 and AutoDockTools4: Automated Docking with Selective Receptor Flexibility. J. of Comput. Chem., 30, 2785-2791.

40. Trott, O.; Olson, A.J. (2010) AutoDock Vina: Improving the speed and accuracy of docking with a new scoring function, efficient optimization, and multithreading. $J$. of Comput Chem., 31, 455-461.

41. Phillips, J.C.; Braun, R.; Wang, W. (2005) Scalable molecular dynamics with NAMD. J. of Comput. Chem., 26, 1781-1802.

42. Pettersen, E.F.; Goddard, T.D.; Huang, C.C., Couch G.S.; Greenblatt, D.M.; Meng, E.C.; Ferrin, T.E. (2004) UCSF Chimera - a visualization system for exploratory research and analysis. J. of comput. Chem., 25, 1605-1612 
43. Salentin, S.; Salentin, S.; Schreiber, S.; Haupt, V.J.; Adasme, M.F.; Schroeder, M. (2015) PLIP: fully automated protein-ligand interaction profiler. Nucleic Acids Res, 43, W443-W447.

44. 1.7.6, V. The PyMOL Molecular Graphics System, Version 1.7.6 Schrödinger, LLC.

45. Sohrabi, C.; Alsafi, Z.; O’Neill, N.; Khan, M.; Kerwan, A.; Al-Jabir, A.; Iosifidis, C.; Agha, R. (2020) World Health Organization declares global emergency: A review of the 2019 novel coronavirus (COVID-19). Inter. J. of Surgery, 76, 71-76.

46. Chen, Y.; Liu, Q.; Guo, D. (2020) Emerging coronaviruses: genome structure, replication, and pathogenesis. J. of Medical Virology, 92, 418-423.

47. Zhou, P.; Lv, J.; Zou, J.; Tian, F.; Shang, Z. (2010) Halogen-water-hydrogen bridges in biomolecules. J. Struct. Biol., 169, 172-182.

48. Vasylyeva, V.; Nayak, S.K., Terraneo, G.; Cavallo, G.; Metrangolo, P.; Resnati G. (2014) Orthogonal halogen and hydrogen bonds involving a peptide bond model. Cryst. Eng. Comm., 16, 8102-8105.

49. Hardegger, L.A., Kuhn, B.; Spinnler, B.; Anselm, L.; Ecabert, R.; Stihle, M.; Gsell, B.; Thoma, R.; Diez, J.; Benz, J.; Plancher, J.M.; Hartmann, G.; Banner, D.W.; Haap, W.; Diederich, F. (2011) Systematic investigation of halogen bonding in proteinligand interactions. Angew. Chem. Int. Ed., 50, 314-318.

50. Vallejos, M.; Auffinger, P.; Ho, P.S. (2012) Halogen interactions in biomolecular crystal structures. Int. Tables Crystallogr. F, 821-826.

51. Maillard M.C.; Hom, R.K.; Benson, T.E.; Moon, J.B.; Mamo, S.; Bienkowski, M.; Tomasselli, A.G.; Woods, D.D.; Prince, D.B.; Paddock, D.J.; Emmons, T.L.; Tucker, J.A.; Dappen, M.S.; Brogley, L.; Thorsett, E.D.; Jewett, N.; Sinha, S.; John, V. (2007) Design, synthesis, and crystal structure of hydroxyethyl secondary amine-based. $J$ Med Chem., 50, 776-781.

52. Iltzsch, M.; Uber, S.S.; Tankersley, K.; Kouni, M.H. (1995) Structure-activity relationship for the binding of nucleoside ligands to adenosine kinase from Toxoplasma gondii. Biochem. Pharmacol., 49, 1501-1512. 
53. Parks, D.J.; Lafrance, L.V.; Calvo, R.R.; Milkiewicz, K.L.; Gupta, V.; Lattanze, J.; Ramachandren, K.; Carver, T.E.; Petrella, E.C.; Cummings, M.D.; Maguire, D.; Grasberger, B.L.; Lu, T. (2005) 1,4-Benzodiazepine-2,5-diones as small molecule antagonists of the HDM2-p53 interaction: Discovery and SAR. Bioorg Med. Chem. Lett., 15, 765-770.

54. Benjahad, A.; Guillemont, J.; Andries, K.; Nguyen, C.H.; Grierson, D.S. (2003) 3-Iodo-4-phenoxypyridinones (IOPY's), a new family of highly potent non-nucleoside inhibitors of HIV-1 reverse transcriptase. Bioorg Med Chem Lett., 13, 4309-4312.

55. Berman, H.M.; Westbrook, J.; Feng, Z.; Gilliland, G,; Bhat, T.N.; Weissig ,H.; Shindyalov, I.N.; Bourne, P.E. (2000) The protein data bank. Nucleic Acids Res., 28, $235-242$.

56. Howard, E.I,; Sanishvili, R.; Cachau, R.E.; Mitschler, A.; Chevrier, B.; Barth P.; Lamour, V.; Zandt, V.M.; Sibley, E.; Bon, C.; Schneider, T.R.; Joachimiak, A.; Podjarny, A. (2004) Ultrahigh resolution drug design I: Details of interactions in human aldose reductase-inhibitor complex at $0.66 \AA$ Aroteins Struct. Funct. Genet., 55, $792-$ 804.

57. Hardegger, L.A.; Kuhn, B.; Spinnler, B.; Anselm, L.; Ecabert, R.; Stihle, M.; Gsell, B.; Thoma R.; Diez, J.; Benz, J.; Plancher, J.M.; Hartmann, G.; Isshiki, Y.; Morikami, K.; Shimma, N.; Haap, W.; Banner, D.W.; Diederich, F. (2011) Halogen bonding at the active sites of human cathepsin L and MEK1 kinase: Efficient interactions in different environments. Chem Med Chem., 6, 2048-2054.

58. Prasad, S.; Wilkinson, J.; Gatzoulis, M.A. (2000) Sildenafil in primary pulmonary hypertension. N. Engl. J. Med., 343, 1342.

59. Xu, Z.; Liu, Z.; Chen, T.; Chen, T.; Wang, Z.; Tian, G.; Shi, J.; Wang, X.; Lu, Y.; Yan, X.; Wang, G.; Jiang, H.; Chen, K.; Wang, S.; Xu, Y.; Shen, J.; Zhu, W. (2011) Utilization of halogen bond in lead optimization: A case study of rational design of potent phosphodiesterase type 5 (PDE5) inhibitors. J. Med. Chem., 54, 5607-5611. 
60. Sung, B.; Hwang, K.; Jeon, Y.; Lee, J.; Heo, Y.; Kim, J.; Moon, J.; Yoon, J.; Hyun, Y.; Kim, E.; Moon J.; Yoon, J.M.; Hyun, Y.L.; Kim, E.; Eum, S.J.; Park, S.Y.; Lee, J.O.; Lee, T.G.; Ro, S.; Cho, J.M. (2003) Structure of the catalytic domain of human phosphodiesterase 5 with bound drug molecules. Nature, 425, 98-102.

61. Raha, K.; Peters, M.B.; Wang, B.; Yu, N.; Wollacott, A.M.; Westerhoff, L.M.; Merz, K.M. (2007) The role of quantum mechanics in structure-based drug design. Drug Discov. Today, 12, 725-731.

62. Mendez, L.; Henriquez, G.; Sirimulla, S.; Narayan M.(2017) Looking back, looking forward at halogen bonding in drug discovery. Molecules 22, 1397-1412.

63. Halvorson, H.O.; Quezada F. (2009) Marine Biotechnology, in Encyclopedia of Ocean Sciences (Second Edition), J.H. Steele, Editor. (2009) Academic Press: Oxford. p. 560-566.

64. Puranik, N.V.; Rani, R.; Singh, V.A.; Tomar, S.; Puntambekar, H.M.; Srivastava, P. (2019) Evaluation of the Antiviral Potential of Halogenated Dihydrorugosaflavonoids and Molecular Modeling with nsP3 Protein of Chikungunya Virus (CHIKV). ACS omega, 4, 20335-20345.

65. Mohareb, R.M.; Abdallah, A.E.M. (2014) New approaches for the synthesis of pyrazole,thiophene, thieno[2,3-b]pyridine, and thiazole derivatives together with their anti-tumore valuations. Med Chem Res., 23, 564-579.

66. Mohareb, R,M.; Wardakhan, W.W.; Hamid, F.I. (2015) Synthesis and cytotoxicity of fused thiophene and pyrazole derivatives derived from 2-N-acetyl-3-cyano-4,5,6,7tetrahydrobenzo[b]thiophene. Med. Chem Res., 24, 2043-2054.

67. Mohareb, R.M.; Zaki, M.Y.; Abas, N.S. (2015) Synthesis, anti-inflammatory and anti-ulcer evaluations of thiazole, thiophene, pyridine and pyran derivatives derived from androstenedione. Steroids, 98, 80-91.

68. Dömling A. (2006) Recent developments in isocyanide based multicomponentreactions in applied chemistry. Chem Rev., 106, 17-89.

69. Rivera, D.G.; León, F. Concepción O, Morales FE, Wessjohann L.A. (2013) A multiple multicomponent approach to chimeric peptide-peptoid Podands. Chem. Eur. J., $19,6417-6428$.

70. Ugi, I.; Werner, B.; Dömling, A. (2003) The chemistry of isocyanides, their multicomponent reactions and their libraries. Molecules, 8, 53-66. 
71. Van-Berkel, S.S.; Bögel, B.G.; Wijdeven. M.A.; Westermann, B.; Rutjes, F.P. (2012) Recent advances in asymmetric isocyanide-based multicomponent reactions. Eur J. Org. Chem., 2012, 3543-3559.

72. Darbarwar, M.; Sundaramurthy, V.(1982) Synthesis of coumarins with 3:4-fused ringsystems and their physiological activity. Synthesis, 337-388.

73. Kemnitzer, W.; Drewe, J.; Jiang, S.; Zhang, H.; Wang, Y.; Zhao, J.; Meerovitch, K. (2004) Discovery of 4-aryl-4H-chromenes as a new series of apoptosis inducers usinga cell-and caspase-based high-through put screening assay. 1.Structure-activity relationships of the 4-aryl group. J. Med. Chem., 47, 6299-6310.

\section{ACKNOWLEDGMENTS}

The University of Tabuk, Kingdom of Saudi Arabia supported Dr.Fahad M. Almutairi with a research Grant 1441- 0190. Fahad M. Almutairi is theDean of Faculty of Science, Tabuk University, Kingdom of Saudi Arabia.

\section{Funding}

This work was financed by the University of Tabuk, Kingdom of Saudi Arabia, Grant 141-0190.

\section{Authors informations}

Fahad M. Almutairi

Department of Biochemistry, Faculty of Science, University of Tabuk, Kingdom of Saudi Arabia

Rafat M. Mohareb

Department of Chemistry, Faculty of Science, Cairo University, Giza, Egypt

Abdo A. Elfiky

Biophysics Department, Faculty of Science, Cairo University, Giza, Egypt

Mahmoud A.A. Mahmoud,

Department of Chemistry, Faculty of Science, University of Tabuk, Kingdom of Saudi Arabia, Tabuk 71491, P. O. Box 741. 
Wagnat W. Wardakhan

Egyptian Drug Authority (EDA) (NODCAR), Cairo, P.O. 29, Egypt

Mervat S. Mohamed

Department of Chemistry, Faculty of Science, Cairo University, Giza, Egypt

Department of Chemistry, Faculty of Science, University of Tabuk, University of Tabuk, Kingdom of Saudi Arabia

Ali Saber Abdelhameed

Department of Pharmaceutical Chemistry, College of pharmacy, King Saud University, Riyadh,11451, Kingdom of Saudi Arabia.

\section{Contributions}

First author F.M. Almutairi collected the data and was responsible about writing this work. Second author R.M. Mohareb the idea of writing this review article, and he performed the literature survey and data research. The third author was responsible about revising the manuscript and writing the references of this work. The fourth and fifth authors M.A. Mahmoud and W.W. Wardakhan responsible for doing experimental work necessary for synthesizing the target molecule and doing the biological screening. The sixth and seventh authors were responsible for the molecular docking of compounds in this work beside writing the biology section.

\section{Corresponding author}

Correspondence to Rafat M. Mohareb

\section{Ethics declarations}

\section{Ethical Approval}

No related ethical issues.

\section{Ethical declaration}


Research involving human participants and/or animals

Not applicable

\section{Informed consent}

Informed consent was obtained from all participants included in the study.

\section{Competing interests}

The authors declare no competing interests.

\section{Consent to participate}

The authors promise that the work described has not been published previously, that it is not under consideration for publication elsewhere, that its publication is approved by all authors and tacitly or explicitly by the responsible authorities where the work was carried out.

\section{Consent to Publish}

The authors promise that if the manuscript is accepted, it will not be published elsewhere in the same form, in English or in any other language, without the written consent of the Publisher. There are no conflicts of interest to declare.

\section{High lights}

*Both of cyclohexan-1,3-dione and dimedone were used to synthesis fused halogen rich heterocyclic compounds.

*The structures of the newly synthesized products were established on the basis of analytical and spectral data.

*The antiviral activities of all novel compounds were studied against human RNA N7MTase (hRNMT) and selected viral N7-MTases such as SARS-CoV nsp14 and Vaccinia D1-D12 complex

* Molecular docking was done for most of the synthesized compounds. 


\section{Figures}

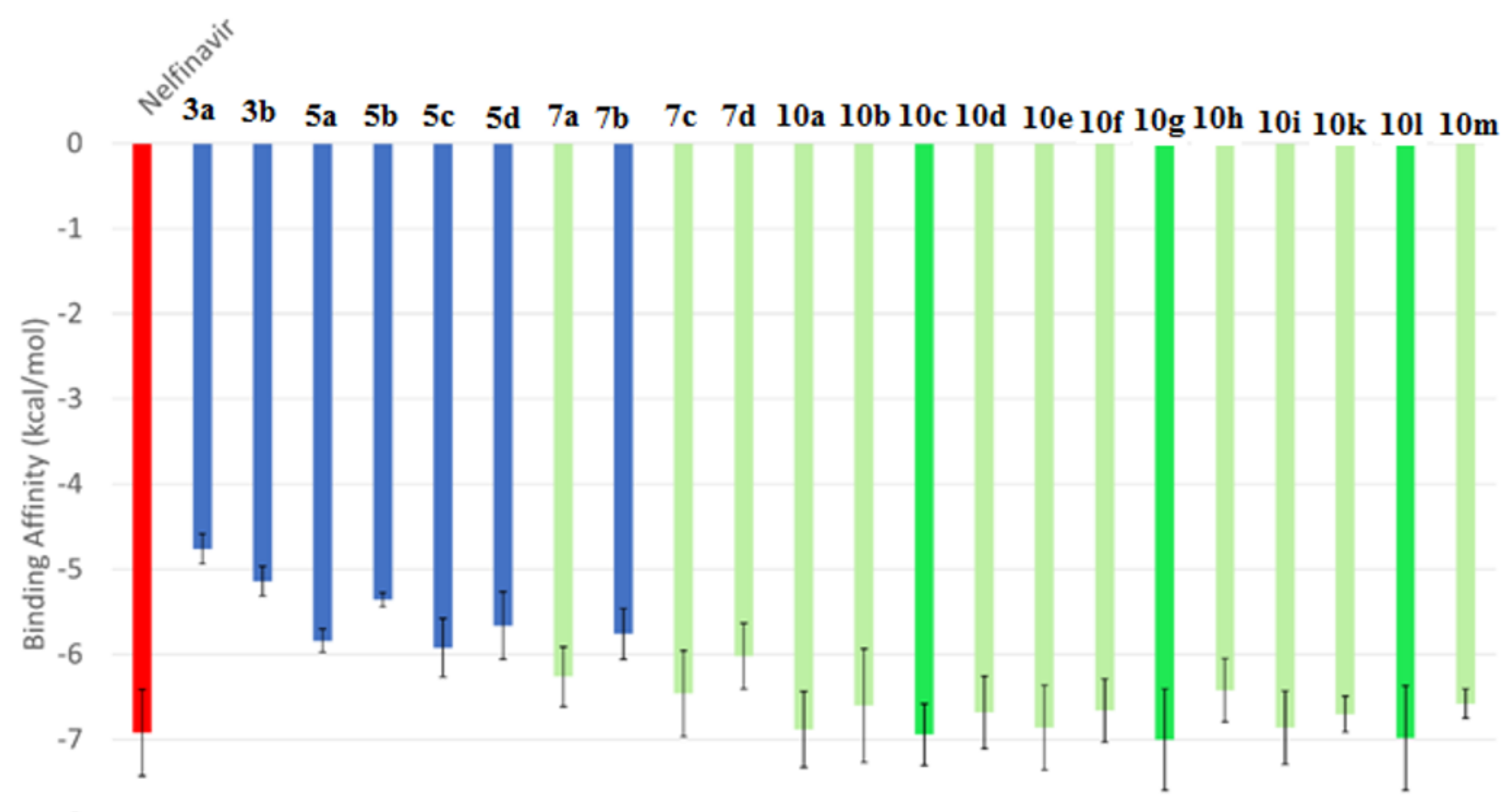

$-8$

Figure 1

The average binding energies $(\mathrm{kcal} / \mathrm{mol})$ of 22 halogenated compounds and Nelfinavir against SARSCoV-2 Mpro active site residue $\mathrm{H} 41$ and $\mathrm{C} 145$. Error bars represent the standard deviation (SD). The halogenated compounds are classified into top compounds (dark green), compounds with comparable binding energywithNelfinavir (light green), and compounds that bind SARS-CoV-2 Mpro with less affinity compared with Nelfinavir (blue), while Nelfinavir is red-colored. 


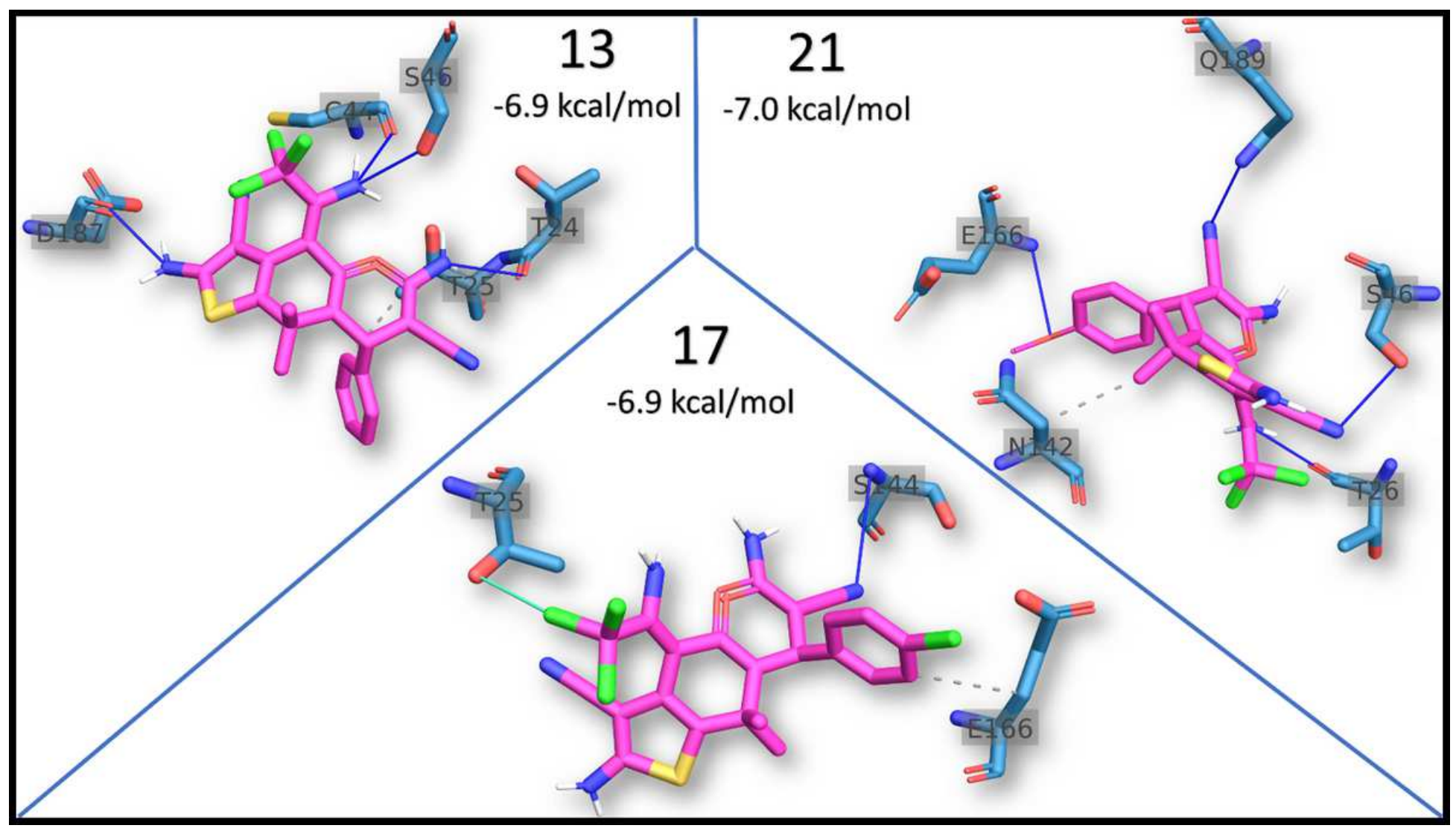

Figure 2

The interaction pattern for the docking of the best three halogenated compounds $(13,17$, and 21$)$ against the active site of SARS-CoV-2 Mpro. H-bonds and the hydrophobic contacts are represented in blue and dashed-gay lines, respectively.Halogen bonds are depicted in green lines. The interacting residues are labeled with their one-letter codes, while the colored sticks represent both the halogenated compounds (magenta) and the interacting residues from SARS-CoV-2 Mpro (blue). 


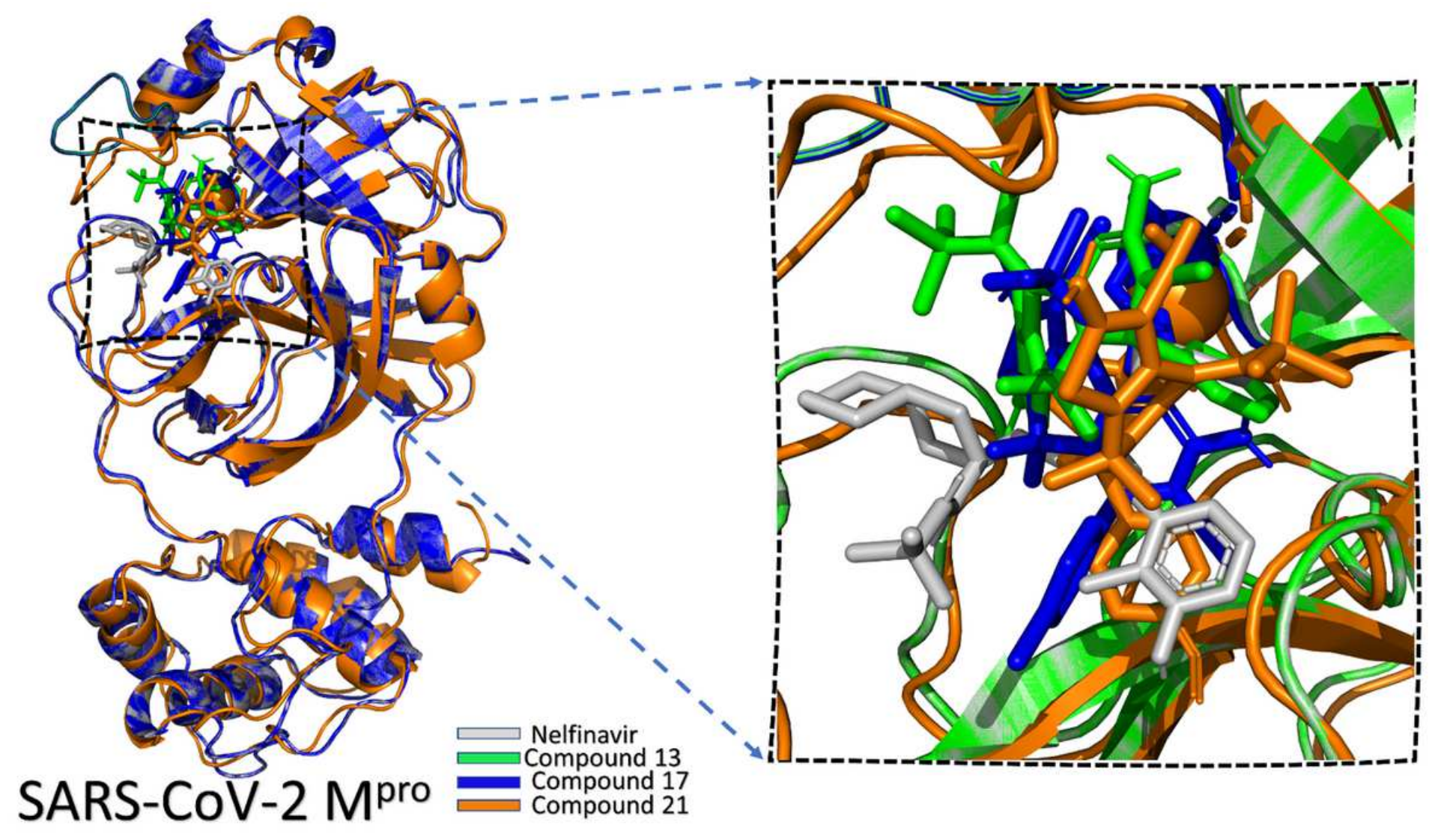

\section{Figure 3}

The superposition of the docking complexes of Nelfinavir (gray), and the compounds $10 \mathrm{c}$ (green), $10 \mathrm{~g}$ (blue), and $10 \mathrm{l}$ (orange) docked into the active site of SARS-CoV-2 Mpro. The enlarged panel is depicted to show how the compounds are lying in the active site cavity of the protein.

\section{Supplementary Files}

This is a list of supplementary files associated with this preprint. Click to download.

- graphicalabstractcmc.docx 